This document is published in:

Journal of Economic Theory (2007), 135 (1), 306-338.

DOI: http://dx.doi.org/10.1016/j.jet.2006.04.007

(C) 2007 Elsevier Inc. 


\title{
Nonlinear pricing with self-control preferences
}

\author{
Susanna Esteban ${ }^{\mathrm{a}, \mathrm{b}, *}$, Eiichi Miyagawa ${ }^{\mathrm{c}}$, Matthew Shum ${ }^{\mathrm{d}}$ \\ a Department of Economics, Pennsylvania State University, 522 Kern Graduate Building, University Park, \\ PA 16802, USA \\ ${ }^{\mathrm{b}}$ Department of Economics, Universidad Carlos III de Madrid, Calle Madrid, 126, 28903 Getafe (Madrid), Spain \\ ${ }^{\mathrm{c}}$ Department of Economics, Columbia University, 420 West 118th Street, New York, NY 10027, USA \\ ${ }^{\mathrm{d}}$ Department of Economics, Johns Hopkins University, 3400 North Charles Street, Baltimore, MD 21218, USA
}

\begin{abstract}
A basic assumption of economics is that consumers choose what they want. However, many consumers find it difficult to stop overeating, overspending, smoking, procrastinating, etc, even though they want to. In reality, consumers have temptation and it is psychologically costly to exercise self-control. To clarify the implications of the existence of temptation and self-control costs, this paper studies a firm's optimal selling strategy exploiting the behavioral features of consumers. We characterize optimal nonlinear pricing schemes for a monopoly when self-control is costly for consumers. Since consumers have a preference for commitment, the firm faces a trade-off between offering a small menu that makes the consumers' self-control easier and offering a large menu that achieves better price discrimination. We show that the optimal menu resembles the one in the standard nonlinear pricing problem with a price ceiling, where the upper bound on prices is determined endogenously by a participation constraint. The ceiling motivates the firm to offer a relatively flat and compact price schedule, serving more consumers with low demand. The characterization also shows that the firm may earn less if consumers have temptation.
\end{abstract}

JEL classification: D11; D42; D82; L11; L12; L15

Keywords: Temptation; Self-control; Commitment; Nonlinear pricing; Price discrimination

\section{Introduction}

Standard economic theories are based on the assumption that consumers choose what they prefer. On the other hand, millions of people are unable to quit smoking even though they want to.

* Corresponding author.

E-mail addresses: sesteban@psu.edu (S. Esteban), em437@columbia.edu (E. Miyagawa), mshum@jhu.edu (M. Shum). 
Many of us also want and fail to stop overeating, overspending, procrastinating, wasting time, etc. It would be safe to say that, for the average consumer, self-control is psychologically costly and incomplete. Traditional economics assumes that self-control is costless. While the assumption is certainly convenient, it is rather extreme and lacks realism. A more natural way to analyze consumers' behavior is to assume that the level of self-control is determined endogenously by its benefits and costs. The simple idea can be captured by a class of utility functions formulated by Gul and Pesendorfer [14]. Using the utility formulation, this paper studies a firm's optimal selling strategy against consumers with temptation and costly self-control.

With standard preferences, a consumer's utility from a set of options depends only on his most preferred option in the set. If $X$ is the choice set and $U$ is the utility function, the utility for the consumer equals $\max _{x \in X} U(x)$. An implication of the standard formulation is that consumers do not mind having more options because additional options can only increase their maximum utility. However, consumers with temptation and costly self-control may not want to have more options since additional options may be tempting and make self-control more difficult.

To capture the idea that "more may be less," Gul and Pesendorfer [14] consider a class of preferences with which the consumer has two utility functions, $U$ and $V$, where $U$ represents the preferences that the consumer would like to commit to- "commitment utility" — and $V$ represents his temptation- "temptation utility." With these utility functions, the consumer chooses an option that maximizes $U+V$, which represents his compromise between the two utility functions. According to this formulation, self-control is exercised to some degree since the realized choice is affected by the commitment utility. Since the choice is also affected by the temptation utility, selfcontrol is incomplete. The level of self-control is determined by the relative cardinal scale of each utility function, which represents the relative bargaining power of temptation and commitment preferences. For example, if the relative scale of $V$ is small, temptation does not have a strong influence over the choice, which means that the consumer has strong willpower.

This modeling is a simple way to capture the intuition that, as Schelling [21] described, a person "behaves like two people, one who wants clean lungs and long life and another who adores tobacco, or one who wants a lean body and another who wants dessert. The two are in a continual contest for control".

For consumers with some willpower, the realized choice does not maximize the temptation utility. The forgone temptation utility, namely, the difference between the realized value of $V$ after self-control and the maximum value of $V$ over the choice set, is considered as a measure of psychological costs of self-control. Gul and Pesendorfer's utility formulation says that a consumer's overall utility from a choice set equals the realized value of $U$ minus self-control costs. That is, if $\hat{x}$ is the realized choice from a set $X$, the consumer's overall utility is given by

$$
\begin{aligned}
W(X) & =U(\hat{x})-\left[\max _{x \in X} V(x)-V(\hat{x})\right] \\
& =\max _{x \in X}[U(x)+V(x)]-\max _{x \in X} V(x) .
\end{aligned}
$$

The second line makes it clear that a larger choice set may be less desirable since it may yield a higher value of $V$. Additional options may be tempting and make self-control more costly.

If self-control is indeed costly for consumers, it should be known to the firms, having implications on their supply behavior. This paper studies a firm's optimal selling strategy in terms of which goods to sell and how much to charge for each of the goods. Does the firm offer many tempting goods to take advantage of consumers' temptation? Or does it have an incentive to simplify its selection to make self-control less painful? How does the incentive affect the degree of price discrimination? 
To address these questions, we build on the standard model of second-degree price discrimination due to Mussa and Rosen [19] and Maskin and Riley [17], where a monopolist sells goods that differ in a single-dimensional quality (or quantity) level $q \in \mathbb{R}_{+}$. The firm does not observe consumers' preferences and therefore uses an indirect price discrimination scheme that relies on consumers' self-selection. The firm's problem is to choose a set of goods $Q \subseteq \mathbb{R}_{+}$to sell and a price function $p: Q \rightarrow \mathbb{R}_{+}$that specifies the price $p(q)$ for each quality level to maximize the firm's expected profit.

A consumer buys from the seller only if the menu $(Q, p)$ satisfies two constraints of individual rationality. One constraint is that after the consumer enters the store, he has an incentive to buy something: the "no-exit" constraint, or what we call ex post IR. The other constraint is that a consumer has an incentive to enter the store: the "entry" constraint or ex ante IR. These constraints are identical under standard preferences: a consumer has an incentive to enter a store if and only if the store sells something worth buying. However, for consumers with costly self-control, a difference appears. Even if a consumer will buy something upon entering the store, he may prefer to stay away from the store if he expects to incur a high level of self-control costs in the store. The entry constraint is what makes the firm's problem distinct from the standard nonlinear pricing problem.

It turns out that the nature of the optimal menu depends sharply on the direction of temptation, which pertains to the relation between $U$ and $V$ for each individual consumer. One important case is where temptation increases the desirability of the goods, in the sense that the marginal value of additional quality relative to the price is higher when the consumer is tempted than when he is not. An example is the case where seeing many high-quality TV sets in a store increases the marginal value of additional quality. In this case, we say that the consumer has upward temptation.

The case of downward temptation is also important, since temptation may increase the desirability of other ways of spending money. For example, consumers often become more frugal when they have to decide and pay. For these consumers, temptation raises the marginal value of money saved. Perhaps, the direction of temptation depends on whether the consumer tends to pay more attention to the good he gets or the amount of money he spends, at the time of decision-making. ${ }^{1}$ There are also domains where downward temptation is the norm. For the participants of weightloss programs, the main temptation is to lose less weight. Temptation lowers their marginal value of weight loss.

We show that if temptation is upward for all consumers, the optimal menu is small (a singleton) and makes self-control unnecessary. Although consumers may differ considerably in their preferences, the firm chooses not to separate them. In contrast, if temptation is downward for all consumers, the optimal menu is large (infinite) and consumers incur positive self-control costs. The optimal menu in this case is identical to the one in the standard model where consumers have standard preferences represented by $U+V$. Therefore, the standard analysis can be used to characterize the optimal menu. Aside from our specific preference formulation, the source of the sharp asymmetry between the cases of upward and downward temptation is the asymmetry that the no-purchase option may be a tempting choice for consumers with downward temptation but not for those with upward temptation.

We also analyze the mixed case where the direction of temptation varies across consumers. The firm's problem in this case turns out to be similar to the one in the standard model with

\footnotetext{
${ }^{1}$ The result of Ameriks et al. [1] suggests not holding a stereotype about the direction of temptation. They conducted a survey asking the respondents how they will deal with a two-period consumption-saving problem. The result is that, surprisingly, $20 \%$ of the respondents reveal temptation to consume less in the first period.
} 
a price ceiling (e.g., [3]). However, there is an important difference. In our problem, the upper bound on prices is not given exogenously but comes from the entry constraint discussed above. Because of the bound, the optimal menu typically induces bunching of high-demand consumers and involves inefficiency for the consumers with the highest demand. The inefficiency means that, for the consumers with the highest marginal valuation, there exists an offer that does not appear in the optimal menu but is preferred by the consumers and generates larger profits for the firm. The firm chooses not to include the Pareto-improving offer since it is so undesirable for the commitment utility that, if it appeared in the menu, the consumers would not enter the store.

By comparing the optimal menu with that in the absence of temptation, we also show that the existence of temptation does not immediately imply that the firm gains from it. Although the firm acts optimally taking advantage of the consumers' temptation, it is theoretically possible that the presence of temptation does not raise the firm's maximum profits. The basic insight is that the consumers' ability to foresee their temptation and self-control can offset the firm's advantage. If temptation may be downward, the firm's profits may be strictly lower in the presence of temptation.

A few papers also study mechanism design for consumers with temptation, but their focus lies on present-biased preferences with hyperbolic discounting. For example, O'Donoghue and Rabin [20] study optimal contracts for motivating a worker to complete a task early when the worker can freely choose when to work. The cost for the worker to complete the task is determined stochastically each period and the worker is unaware of his present bias (he is "naive"). The optimal contract for the firm may exhibit a "deadline" in the sense that the punishment for a delay becomes more severe after a certain date. Gilpatric [13] studies optimal contracts for motivating a worker to exert effort at a fixed point in time. A present bias is relevant since the worker is rewarded after his work with a time lag. The paper shows that the firm may want to offer a contract such that the workers who are aware of their present bias will walk away. DellaVigna and Malmendier [7] study optimal two-part tariffs, in a complete information setup, for a service whose costs and benefits accrue to the consumer in different points in time. A present bias is relevant since consumers have to pay a lump-sum fee before using the service. The paper shows that the optimal usage fee is below (respectively above) the usage cost to the firm if the benefits accrue to the consumers later (respectively earlier) than the costs. ${ }^{2}$

The current paper is not concerned with present-bias. Present-bias is certainly in the background as a major reason for temptation. But our focus is on a firm's pricing strategy as to which goods to sell and at what prices. We focus on how temptation affects consumers' preferences over different products. As suggested above, the effect of temptation on how the consumer values each of the products appears to vary with the context and individual. In this paper, we clarify in what way the seller's optimal strategy depends on the direction of the consumers' temptation. We are also the first to study a firm's strategy when self-control is costly.

There is also an empirical literature that tests for the presence of preference reversals using pricing data. DellaVigna and Malmendier [8] look for this evidence in health club membership data and find that the data can be better explained with the behavior of time-inconsistent consumers. Miravete [18] tests for the presence of irrational behavior in calling plan choices (where the popularity of flat rate plans has been viewed as evidence of irrationality). He finds the data to be consistent with a model of learning and rational behavior. Wertenbroch [25] finds evidence of

\footnotetext{
${ }^{2}$ A few other papers that use Gul-Pesendorfer preferences include the following. Krusell et al. [15] consider a neoclassical growth model with self-control preferences. Krusell et al. [16] analyze a general-equilibrium asset pricing model where consumers have temptation to save. DeJong and Ripoll [5] analyze whether self-control preferences in an asset pricing model can explain the behavior of stock prices.
} 
lower price-elasticity for goods with delayed negative effects (e.g., cigarettes), which suggests that consumers ration the purchase of these goods to control their impulse consumption.

\section{Menu}

The model is a standard model of nonlinear pricing, as in Mussa and Rosen [19] and Maskin and Riley [17], except that consumers have temptation and costly self-control. There is a monopolist selling a set of goods. The goods are indexed by a scaler $q \in \mathbb{R}_{+}$, which represents, as usual, either quantity or quality. We will refer to it as quality. The good of quality $q=0$ is the equivalent of nothing.

Each consumer is interested in buying at most one unit of one good. An offer from the seller is defined as a pair $(q, t) \in \mathbb{R}_{+}^{2}$. With the offer available, a consumer can buy good $q$ for a total charge of $t$. A menu is a compact subset of offers $M \subseteq \mathbb{R}_{+}^{2}$ such that $(0,0) \in M$. The restriction $(0,0) \in M$ means that a consumer who does not make a purchase does not have to pay. In what follows, we sometimes omit $(0,0)$ in the description of a menu: by $M=\left\{(q, t),\left(q^{\prime}, t^{\prime}\right), \ldots\right\}$, we mean $M=\left\{(0,0),(q, t),\left(q^{\prime}, t^{\prime}\right), \ldots\right\}$.

\section{Consumers' preferences}

Consumers have preferences over menus. Their preferences differ in the way in which they are tempted and are parameterized by a number $\gamma \in \mathbb{R}_{+}$. We assume that $\gamma$ is the consumer's private information and is distributed in the population according to a distribution function $F$. The associated density function is $f$, which has support $\Gamma \equiv[a, b]$, where $a$ and $b$ are finite.

The utility function of a consumer of type $\gamma$ is given by

$$
W_{\gamma}(M) \equiv \max _{(q, t) \in M}\left[U(q, t)+V_{\gamma}(q, t)\right]-\max _{(q, t) \in M} V_{\gamma}(q, t),
$$

where $U$ and $V_{\gamma}$ are functions from $\mathbb{R}_{+}^{2}$ into $\mathbb{R}$. The function $U$ represents the preferences that the consumer would like to commit to, while $V_{\gamma}$ represents his temptation. We call $U$ the commitment utility and $V_{\gamma}$ the temptation utility. The actual choice of the consumer is the offer that maximizes $U+V_{\gamma}$, which is interpreted as the compromise between the two utility functions. Since the consumer's choice is influenced by $U$, the consumer exercises self-control to some degree. Since $V$ also influences the choice, self-control is not complete. The strength of self-control-the "bargaining power" of each utility function-is determined by the relative scale of each utility function. If the scale of $U$ is large relative to that of $V$, the consumer has strong willpower. ${ }^{3}$

The choice that maximizes $V_{\gamma}$ represents the most tempting offer in the menu. The role of the maximization can be seen by rearranging (1) into

$$
W_{\gamma}(M)=U(\hat{x})-\left[\max _{x \in M} V_{\gamma}(x)-V_{\gamma}(\hat{x})\right],
$$

where $\hat{x}$ is a maximizer of $U+V_{\gamma}$. The term in square brackets represents the amount of temptation utility that the consumer forgoes as a result of self-control. The consumer is tempted to maximize $V_{\gamma}$, but he ends up with $\hat{x}$ as a result of self-control. The forgone utility of $V_{\gamma}$ therefore quantifies

\footnotetext{
${ }^{3}$ Similar two-self formulations of self-control have been considered by Winston [26], Thaler and Shefrin [24], and Shefrin and Thaler [23]. More recent papers that suggest similar formulations are Fudenberg and Levine [11] and Chatterjee and Krishna [4]. A generalization of Gul-Pesendorfer preferences is proposed by Dekel et al. [6].
} 


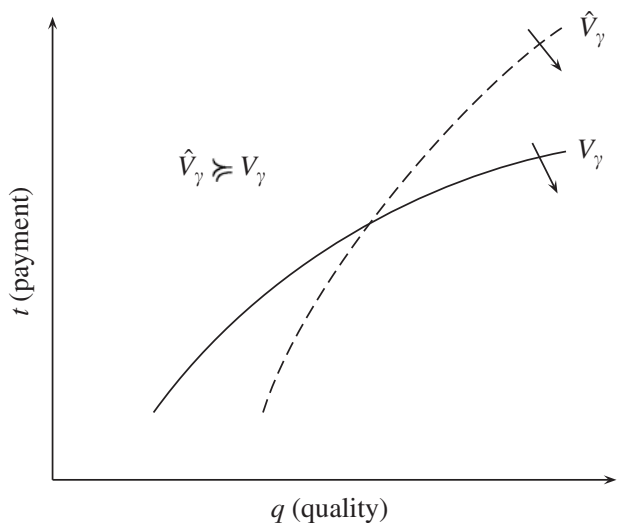

Fig. 1. A representative indifference curve for each utility function.

the cost of self-control. ${ }^{4}$ With the definition, the consumer's overall utility equals the commitment utility from his actual choice minus the self-control cost. Since self-control costs may be higher for larger menus, consumers may prefer smaller menus, exhibiting a preference for commitment. But, of course, consumers may prefer a larger menu. A larger menu may be preferable if additional items yield a higher value of $U(\hat{x})$.

The first maximization problem in (1) is called the ex post problem or the $U+V_{\gamma}$ problem. The maximum value of $U+V_{\gamma}$ is called the ex post utility from $M$ and denoted as $w_{\gamma}(M)$. The overall utility from the menu, i.e., $W_{\gamma}(M)$, is called the ex ante utility from $M$.

We place the following standard assumptions on $U$ and $V$ :

Assumption 1. The functions $U$ and $V_{\gamma}$ are continuous, strictly increasing in $q$, strictly decreasing in $t$, quasi-concave, and satisfy $U(0,0)=V_{\gamma}(0,0)=0$; and, the process $\left\{V_{\gamma}\right\}_{\gamma \in \mathbb{R}_{+}}$, viewed as a function of $(q, t, \gamma)$, is continuous.

Given two utility functions $V_{\gamma}$ and $\hat{V}_{\gamma}$ (defined over $\mathbb{R}_{+}^{2}$ ), we write $\hat{V}_{\gamma} \succcurlyeq V_{\gamma}$ if the marginal value of quality is weakly higher for $\hat{V}_{\gamma}$ than for $V_{\gamma}$ at any point $(q, t) \in \mathbb{R}_{+}^{2}$. Graphically, the indifference curve of $\hat{V}_{\gamma}$ is at least as steep as that of $V_{\gamma}$ when the first (respectively, second) argument is measured on the horizontal (respectively, vertical) axis. Fig. 1 depicts representative indifference curves. Formally, $\hat{V}_{\gamma} \succcurlyeq V_{\gamma}$ if and only if, for all $(q, t),\left(q^{\prime}, t^{\prime}\right) \in \mathbb{R}_{+}^{2}$ such that $q^{\prime}>q$,

$$
V_{\gamma}\left(q^{\prime}, t^{\prime}\right) \geqslant V_{\gamma}(q, t) \text { implies } \hat{V}_{\gamma}\left(q^{\prime}, t^{\prime}\right) \geqslant \hat{V}_{\gamma}(q, t)
$$

and

$$
V_{\gamma}\left(q^{\prime}, t^{\prime}\right)>V_{\gamma}(q, t) \text { implies } \hat{V}_{\gamma}\left(q^{\prime}, t^{\prime}\right)>\hat{V}_{\gamma}(q, t) .
$$

If $\hat{V}_{\gamma} \succcurlyeq V_{\gamma}$ and $\hat{V}_{\gamma} \preccurlyeq V_{\gamma}$, the two functions induce the same indifference map, which is denoted as $\hat{V}_{\gamma} \sim V_{\gamma}$.

\footnotetext{
${ }^{4}$ For different notions of self-control costs, see, e.g., Thaler and Shefrin [24] and Fudenberg and Levine [11].
} 
We also write $\hat{V}_{\gamma} \succ V_{\gamma}$ if the indifference curve of $\hat{V}_{\gamma}$ is strictly steeper than that of $V_{\gamma}$ at any point $(q, t)$. Formally, $\hat{V}_{\gamma} \succ V_{\gamma}$ if and only if, for all $(q, t),\left(q^{\prime}, t^{\prime}\right) \in \mathbb{R}_{+}^{2}$ such that $q^{\prime}>q$,

$$
V_{\gamma}\left(q^{\prime}, t^{\prime}\right) \geqslant V_{\gamma}(q, t) \text { implies } \hat{V}_{\gamma}\left(q^{\prime}, t^{\prime}\right)>\hat{V}_{\gamma}(q, t)
$$

which is the case drawn in Fig. 1.

We place the following assumptions.

Assumption 2. For all $\gamma, \gamma^{\prime}$, if $\gamma^{\prime}>\gamma$, then $V_{\gamma^{\prime}} \succ V_{\gamma}$.

Assumption 3. There exists a type $\gamma^{*} \in \mathbb{R}_{+}$such that

$$
\begin{array}{ll}
V_{\gamma} \preccurlyeq U & \text { if } \gamma \leqslant \gamma^{*}, \\
V_{\gamma} \succcurlyeq U & \text { if } \gamma \geqslant \gamma^{*} .
\end{array}
$$

Assumption 4. For any pair of types $\gamma, \theta \in \mathbb{R}_{+}$such that $\theta>\gamma$ and any pair of offers $x, y \in \mathbb{R}_{+}^{2}$ such that $U(x)+V_{\gamma}(x)<U(y)+V_{\gamma}(y)$, there exists $z \in \mathbb{R}_{+}^{2}$ such that

$$
U(z)+V_{\gamma}(z)=U(x)+V_{\gamma}(x) \text { and } \quad U(z)+V_{\theta}(z)=U(y)+V_{\theta}(y) .
$$

Assumption 2 is a single-crossing property, saying that the indifference curves of $V_{\gamma}$ are steeper for higher types.

Assumption 3 says that there exists a critical type $\gamma^{*}$ at which the direction of temptation switches. For consumers of type $\gamma>\gamma^{*}$, the marginal value of additional quality is higher when they are tempted than when they are not: $V_{\gamma} \succ U+V_{\gamma} \succ U$. These consumers are tempted by goods of higher quality, and we say that they have upward temptation.

For consumers of type $\gamma<\gamma^{*}$, on the other hand, temptation lowers the marginal value of quality: $V_{\gamma} \prec U+V_{\gamma} \prec U$. These consumers have downward temptation. For these consumers, temptation raises the marginal value of money saved. For example, consumers may become more frugal when it is time to decide and pay. Perhaps, the marginal value of money increases temporarily as the consumer's attention gets focused on the amount of expenditure. In contrast, temptation may be upward if, at the time of decision-making, the consumer pays more attention to the good he gets than the amount he pays.

There are also situations in which temptation is normally downward. For example, for participants of a weight loss program (e.g., Weight Watchers), temptation lowers the marginal value of additional weight loss. For them, temptation raises the marginal value of eating or not exercising. This is a situation where the good is desirable in the long run but less so in the short run. Similar examples can be found for people who want to study, work, quit smoking, etc.

Consumers of the threshold type $\gamma^{*}$ do not have a temptation problem since their temptation preferences coincide with their commitment preferences. Indeed, Assumption 3 implies $V_{\gamma^{*}} \sim U$.

Assumption 4 is a technical condition saying that any pair of indifference curves, one for $U+V_{\gamma}$ and the other for $U+V_{\theta}$, should cross somewhere, except when the steeper one lies entirely above the flatter one. Intuitively speaking, the assumption says that if the curves do not cross, they do cross in $\mathbb{R}^{2} \backslash \mathbb{R}_{+}^{2}$ if we extend the curves naturally from $\mathbb{R}_{+}^{2}$ to $\mathbb{R}^{2}$. 


\section{The seller's problem}

Let $C: \mathbb{R}_{+} \rightarrow \mathbb{R}_{+}$be the per-consumer cost function, where $C(q)$ denotes the cost of offering good $q$ to a consumer. For any offer $x=(q, t)$, let $\pi(x) \equiv t-C(q)$ denote the per-consumer profit from the offer. The cost function is assumed to satisfy the following standard assumptions.

Assumption 5. $C$ is strictly increasing, convex, and satisfies $C(0)=0$. Further, there exists a unique solution $x^{*} \gg(0,0)$ that maximizes $\pi(x)$ subject to $U(x) \geqslant 0$.

An assignment function for a given menu $M$ is a function $x(\cdot)=(q(\cdot), t(\cdot)): \Gamma \rightarrow M$ that assigns to each type $\gamma$ an offer in the menu. As usual, the interpretation is that $x(\gamma)$ is the offer that the type $\gamma$ is expected to choose. Since $M$ contains $(0,0)$, we allow for $x(\gamma)=(0,0)$.

Consumers have the freedom of not choosing from $M$. If $M$ represents a store, this means that consumers have the option of not entering the store. If a consumer does not choose from $M$, he is thought to be choosing from $M_{0}=\{(0,0)\}$. Consumers of type $\gamma$ have an incentive to choose from $M$ only if $W_{\gamma}(M) \geqslant 0$, in which case $M$ is said to satisfy ex ante IR for type $\gamma$. In what follows, we let $E \subseteq \Gamma$ denote the set of types who choose from $M$. Since consumers $\gamma \notin E$ choose $M_{0}=\{(0,0)\}$, the assignment function has to satisfy $x(\gamma)=(0,0)$ for all $\gamma \notin E$.

A schedule is an ordered list $(M, x, E)$, where $M$ is a menu, $x: \Gamma \rightarrow M$ is an assignment function, and $E \subseteq \Gamma$. The firm's problem is to choose a schedule $(M, x, E)$ that maximizes

$$
\int[t(\gamma)-C(q(\gamma))] d F(\gamma)
$$

subject to that, for all $\gamma \in E$,

$$
\begin{aligned}
& W_{\gamma}(M) \geqslant 0, \\
& U(x(\gamma))+V_{\gamma}(x(\gamma)) \geqslant 0, \\
& U(x(\gamma))+V_{\gamma}(x(\gamma)) \geqslant U(y)+V_{\gamma}(y) \quad \text { for all } y \in M,
\end{aligned}
$$

(ex ante IR)

and, for all $\gamma \notin E$,

$$
\begin{aligned}
& W_{\gamma}(M) \leqslant 0, \\
& x(\gamma)=(0,0) .
\end{aligned}
$$

The ex post IR and IC conditions together imply that, for each type who chooses $M, x(\gamma)$ is an optimal choice for $U+V_{\gamma}$. On the other hand, for the consumers who stay away from $M, x(\gamma)$ equals $(0,0)$ and is not required to satisfy ex post IC with respect to $M .^{5}$

A schedule that satisfies all the constraints is called a feasible schedule. If a feasible schedule $(M, x, E)$ solves the maximization problem, it is an optimal schedule and $M$ is an optimal menu. We only consider the problem for which the monopolist can earn strictly positive profits.

The ex post IR and IC conditions are independent of the consumers' $V$ problem (their second maximization problem). The $V$ problem affects the monopolist's problem only because it affects the consumers' ex ante utility levels and thereby whether they have an incentive to enter the store.

If the consumers' entry decisions are ignored, i.e., if all consumers are assumed to choose from $M$, then the monopolist's problem reduces to the standard nonlinear pricing problem of Mussa

\footnotetext{
${ }^{5}$ Consumers for whom $W_{\gamma}(M)=0$ are indifferent about their entry decision. We assume that their decisions are resolved in the way the firm prefers.
} 
and Rosen where the utility function of type- $\gamma$ consumers is $U+V_{\gamma}$. In the reduced problem, ex post IR and IC have to be satisfied for each type, while ex ante IR is not required. This problem serves as a useful benchmark to the monopolist's problem and is referred to as the ex post selling problem or the standard problem with utility functions $U+V_{\gamma}$. Since the ex post selling problem is a standard nonlinear pricing problem, its solution satisfies well-known properties: an optimal menu consists of a continuum of offers, leaves no surplus to the lowest type (ex post IR holds with equality for type $a$ ), and achieves social efficiency for the highest type (the indifference curve of $U+V_{\gamma}$ for type $b$ is tangent to the isoprofit curve). ${ }^{6}$

Another useful benchmark is the case without temptation, i.e., $V_{\gamma} \sim U$ for all $\gamma$. If there is no temptation, the self-control cost is zero. Since the $U$ function is common across consumers, the firm will offer the menu $M=\left\{x^{*}\right\}$, where $x^{*}$ solves

$$
\max \pi(x) \quad \text { subject to } U(x) \geqslant 0 .
$$

By Assumption 5, $x^{*}$ is uniquely defined.

\section{The case of complete information}

Before we characterize optimal schedules, we clarify the seller's optimal strategy when each consumer's type can be observed by the seller. Consider a consumer of type $\gamma$ and suppose that the seller knows the consumer's type. Then the seller can offer a personalized menu $M$ to the consumer. If the seller wants the consumer to choose $x \in M$, then $(M, x)$ need to satisfy

$$
\begin{aligned}
& U(x)+V_{\gamma}(x) \geqslant 0, \\
& U(x)+V_{\gamma}(x) \geqslant U(y)+V_{\gamma}(y) \text { for all } y \in M, \\
& W_{\gamma}(M) \geqslant 0 .
\end{aligned}
$$

But, if a pair $(M, x)$ satisfies (2)-(4), so does $(\{x\}, x)$. That is, the seller does not gain by adding any other item to the menu since the added items can only raise the consumers' self-control cost. Therefore, the constraints reduce to (2) and (4), where the latter is equivalent to

$$
U(x)+V_{\gamma}(x)-\max \left\{0, V_{\gamma}(x)\right\} \geqslant 0 .
$$

Since (5) implies (2), the seller's problem reduces to maximizing $\pi(x)$ subject to (5). We denote its solution by $x_{\gamma}^{*}$ and call it the perfect discrimination offer for consumers of type $\gamma$.

The exact characterization of the perfect discrimination offers depends on the direction of the consumer's temptation. For all consumers with upward temptation, (2)-which is redundant but has to be satisfied-implies $V_{\gamma}(x) \geqslant 0$. Thus (5) reduces to $U(x) \geqslant 0$. Conversely, any $x$ such that $U(x) \geqslant 0$ satisfies $V_{\gamma}(x) \geqslant 0$ and hence (5). Therefore, $x_{\gamma}^{*}$ is a solution to maximizing $\pi(x)$ subject to $U(x)=0$, which implies $x_{\gamma}^{*}=x^{*}$ for all $\gamma \geqslant \gamma^{*}$. The personalized menu $M=\left\{x^{*}\right\}$ does not impose a self-control cost on the consumer.

For consumers with downward temptation, on the other hand, any $x$ such that $U(x)+V_{\gamma}(x)=0$ satisfies $V_{\gamma}(x) \leqslant 0$ and hence (5). Therefore, $x_{\gamma}^{*}$ maximizes $\pi(x)$ subject to $U(x)+V_{\gamma}(x)=0$. The personalized menu $\left\{x_{\gamma}^{*}\right\}$ imposes a positive self-control cost of $V_{\gamma}(0,0)-V_{\gamma}\left(x_{\gamma}^{*}\right)>0$. The consumer is tempted by $(0,0)$.

\footnotetext{
${ }^{6}$ See, for example, Fudenberg and Tirole [12, Chapter 7].
} 


\section{Ex Ante IR}

We now return to the case of incomplete information and begin the characterization of the optimal schedule. We start by characterizing the ex ante IR constraint since the constraint is what distinguishes our pricing problem from the standard one.

We first show that, for any menu, all consumers for whom ex ante IR is satisfied receive a non-negative utility of $U$.

Lemma 1. Suppose that a menu $M$ satisfies ex ante IR for a type $\gamma$. Then, for any offer $x \in M$ solving the $U+V_{\gamma}$ problem, $U(x) \geqslant 0$.

Proof. Since $M$ satisfies ex ante IR for $\gamma$ and $x$ maximizes $U+V_{\gamma}$,

$$
0 \leqslant W_{\gamma}(M)=U(x)+V_{\gamma}(x)-\max _{y \in M} V_{\gamma}(y) \leqslant U(x) .
$$

In words, the ex ante utility equals the utility in $U$ minus the self-control cost. Since the selfcontrol cost is non-negative, a necessary condition for the ex ante utility to be non-negative is that the utility $U$ be non-negative.

On the other hand, for consumers for whom ex ante IR is violated, $x(\gamma)=(0,0)$ and hence $U(x(\gamma))=0$. This yields the next corollary.

Corollary 1. For any feasible schedule $(M, x, E)$ and any type $\gamma$, we have $U(x(\gamma)) \geqslant 0$.

The corollary says that offers $x$ such that $U(x)<0$ are never chosen by any consumer.

The following lemma is a converse of Lemma 1 and Corollary 1, stating that if all offers in a menu leave non-negative $U$ surplus, the menu necessarily satisfies ex ante IR for all consumers.

Lemma 2. If a menu $M$ satisfies $U(x) \geqslant 0$ for all $x \in M$, ex ante IR is satisfied for all consumers.

Proof. Let $M$ be a menu such that $U(x) \geqslant 0$ for all $x \in M$, and let $\gamma$ be any type. Let $y \in M$ be an offer that maximizes $V_{\gamma}$. Then

$$
W_{\gamma}(M)=\max _{x \in M}\left[U(x)+V_{\gamma}(x)\right]-V_{\gamma}(y) \geqslant U(y)+V_{\gamma}(y)-V_{\gamma}(y)=U(y) \geqslant 0 .
$$

In words, $y$ is a most tempting offer in the menu. Upon entering the store, the consumer has an option of succumbing to the temptation completely choosing $y$, in which case his overall utility is $U(y)$ since the self-control cost is zero. By ex post IC, he attains at least this level of ex ante utility. If all offers in the menu yield non-negative utility in $U$, then $U(y) \geqslant 0$ and so the ex ante utility is also non-negative.

Corollary 1 says that all offers that are chosen by some consumers give non-negative $U$ surplus, while Lemma 2 says if all offers in the menu give non-negative $U$ surplus then ex ante IR is satisfied. These results together imply that if a feasible schedule $(M, x, E)$ is not "decorated" in the sense that every item in the menu is chosen by some consumer, then ex ante IR is satisfied for all types. Formally,

Definition. A feasible schedule $(M, x, E)$ is undecorated if $M=\operatorname{closure}\{x(\gamma): \gamma \in \Gamma\}$. 
Then Corollary 1 and Lemma 2 imply

Corollary 2. If a feasible schedule $(M, x, E)$ is undecorated, then $U(x) \geqslant 0$ for all $x \in M$ and $M$ satisfies ex ante IR for all types.

To search for an optimal menu, we can focus on undecorated menus. Indeed, if a menu is decorated, then by removing the offers that are not chosen by any consumer, the firm can make the menu less tempting and raise the consumers' ex ante utility. Formally,

Proposition 1. For any feasible schedule $(M, x, E)$, there exists a feasible schedule $\left(M^{\prime}, x^{\prime}, E^{\prime}\right)$ that generates at least as large profits as $(M, x, E)$ and such that

1. $M^{\prime}=\operatorname{closure}\left\{x^{\prime}(\gamma): \gamma \in \Gamma\right\}$ (i.e., the schedule is undecorated),

2. $U(y) \geqslant 0$ for all $y \in M^{\prime}$ (i.e., all offers in the menu generate non-negative $U$ surplus),

3. $E^{\prime}=\Gamma$ (i.e., all types enter the store),

4. $\pi(x) \geqslant 0$ for all $x \in M^{\prime}$ (i.e., all offers in the menu generate non-negative profits).

The proof is relegated to the Appendix, which also contains all other proofs omitted from the text. The proposition motivates us to introduce the following definition.

Definition. An optimal schedule $(M, x, E)$ is regular if it satisfies properties $1-4$ in Proposition 1.

Proposition 1 then implies that if an optimal schedule exists, there exists one that is regular.

\section{Upward temptation}

To characterize optimal schedules, we first consider the case where all consumers have upward temptation, i.e., $\gamma \geqslant \gamma^{*}$ for all $\gamma \in \Gamma$. In this case, we show that the seller can extract the entire ex ante surplus of the consumers by offering a singleton menu given by $M=\left\{x^{*}\right\}$.

Proposition 2. If all consumers have upward temptation, then $M=\left\{x^{*}\right\}$ is an optimal menu and extracts each consumer's entire ex ante surplus.

Proof. Suppose the monopolist offers $\left\{x^{*}\right\}$. Since $U\left(x^{*}\right) \geqslant 0$, Lemma 2 implies that the menu satisfies ex ante IR for all types. The remaining question is whether all consumers will choose $x^{*}$ over $(0,0)$. Since the temptation is upward for all consumers, $U\left(x^{*}\right) \geqslant 0$ implies $U\left(x^{*}\right)+$ $V_{\gamma}\left(x^{*}\right) \geqslant 0$ and hence $x^{*}$ satisfies ex post IR.

Although $(0,0)$ is in the menu, it does not tempt consumers whose temptation is upward. As a result, the menu $\left\{x^{*}\right\}$ eliminates self-control costs and enables the seller to extract the entire ex ante surplus of consumers. Ex post, consumers of type $\gamma>\gamma^{*}$ obtain a positive surplus equal to $U\left(x^{*}\right)+V_{\gamma}\left(x^{*}\right)=V_{\gamma}\left(x^{*}\right)>0$, but the seller cannot extract this surplus since doing so makes the consumer's ex ante utility negative.

\section{Downward temptation}

We now consider the case where all consumers have preferences with downward temptation: $\gamma \leqslant \gamma^{*}$ for all $\gamma \in \Gamma$. The following proposition shows that for consumers with downward temptation, ex ante IR never binds. 
Proposition 3. For all consumers with downward temptation, any menu $M$ satisfies ex ante IR, i.e., $W_{\gamma}(M) \geqslant 0$.

Proof. Consider a consumer with downward temptation and consider any menu $M$. Let $y \in M$ be an offer that maximizes $V_{\gamma}$. Then the ex ante utility from the menu is

$$
W_{\gamma}(M)=\max _{x \in M}\left[U(x)+V_{\gamma}(x)\right]-V_{\gamma}(y) \geqslant U(y)+V_{\gamma}(y)-V_{\gamma}(y)=U(y) .
$$

Since $y$ maximizes $V_{\gamma}$ when $(0,0)$ is also available, $V_{\gamma}(y) \geqslant 0$. Since the temptation is downward, this implies $U(y) \geqslant 0$. Therefore, (6) implies $W_{\gamma}(M) \geqslant 0$.

A corollary is that if all consumers have downward temptation, we can ignore the ex ante IR constraint. As a result, the monopolist's problem reduces to the standard problem where each type's utility function is simply $U+V_{\gamma}$. Therefore, the optimal menu can be identified by the standard technique of nonlinear pricing.

Corollary 3. Suppose that all consumers have downward temptation, i.e., $\gamma \leqslant \gamma^{*}$ for all $\gamma \in \Gamma$. Then $(M, x, E)$ is an optimal schedule if and only if $(M, x)$ is an optimal schedule in the ex post selling problem.

\section{Mixed case}

This section studies the case where some consumers have downward temptation and others have upward temptation $\left(a<\gamma^{*}<b\right)$. We divide our analysis into two sections. The first section clarifies the properties of optimal menus in general environments. Section 9.2 gives sharper characterizations by assuming quasi-linear and differentiable preferences.

\subsection{General properties of optimal menus}

The first property of optimal menus is that ex post IR binds for the lowest consumer type.

Proposition 4. For any regular optimal schedule, $U(x(a))+V_{a}(x(a))=0$.

This is not a trivial result in our context. In the standard problem, if ex post IR is not binding for the lowest type, one argues that the firm can simply shift the entire price schedule by a constant $\varepsilon>0$ without changing consumers' participation decisions. In the present problem, however, the same argument does not work, since if the entire menu is shifted, the new menu may no longer satisfy ex ante IR, which requires $U(x(\gamma)) \geqslant 0$. Therefore some consumers may choose to stay away from the modified menu, which makes the total effect on profits ambiguous. We deal with the problem by raising prices across quality levels in a non-uniform way. Specifically, we determine the price increase for each quality level so that the new price generates the same per-person profit as the lowest quality sold. If the price increment so defined is negative, we keep the initial price. We then remove the offers such that $U(x)<0$. The deletion does not pose a problem since all remaining offers are at least as profitable as any of the deleted offers. There is a technical complication since the initial menu may not be a connected set, i.e., the quality function $q(\gamma)$ may have discontinuous jumps. The proof deals with the issue by first filling the "holes" in the menu to obtain a single connected set. 
It should be noted that the conclusion of Proposition 4 is not true if all consumers have upward temptation. Indeed, the optimal menu in Proposition 2 gives a strictly positive ex post utility to all types $\gamma>\gamma^{*}$. If $a>\gamma^{*}$, therefore, the lowest type gets a positive ex post utility in the optimal schedule.

The next result says that the right end of the tariff gives no surplus in terms of $U$ if the highest type has a sufficiently high marginal value for quality.

Proposition 5. Suppose that, for all offers $x \in \mathbb{R}_{+}^{2}$ such that $\pi(x) \geqslant 0$ and $U(x) \geqslant 0$, there exists a type $\gamma \in \Gamma$ and an offer $y \gg x$ such that

$$
\pi(y)>\pi(x) \text { and } U(y)+V_{\gamma}(y)>U(x)+V_{\gamma}(x) .
$$

Then, for any regular optimal schedule, $U(x(b))=0$.

To get the intuition, suppose the optimal price schedule ends before reaching the $U=0$ curve. Then at $x(b)$, the isoprofit curve and type $b$ 's indifference curve generate a lens-shaped area in the north-east consisting of offers that both the firm and consumers of type $b$ prefer to $x(b)$. Choose any offer $y$ in the interior of this lens and below the $U=0$ curve, and add it to the menu. Since consumers $\gamma$ close to $b$ choose the added offer, the modified menu generates more profits.

We can also show that the price schedule reaches the $U=0$ curve to the right of $x^{*}$.

Proposition 6. Assume the same condition as Proposition 5. Then, for any regular optimal schedule, $x(b) \geqslant x^{*}$. If $U(q, t)=u(q)-t$, and $u$ and $C$ are differentiable, then $u^{\prime}(q(b)) \leqslant C^{\prime}(q(b))$.

If the menu reaches the $U=0$ curve to the left of $x^{*}$, the firm can raise profits by adding $x^{*}$ to the menu. Indeed, $x^{*}$ will then be chosen by some of the high-type consumers and yield more profits than any other offer in the initial menu. Since $x^{*}$ satisfies $U \geqslant 0$, its addition preserves ex ante IR.

\subsection{Differential approach}

To obtain sharper characterizations of optimal menus, we now assume that preferences are quasi-linear and differentiable. We consider the following specification:

$$
\begin{aligned}
U(q, t) & =u(q)-t, \\
V_{\gamma}(q, t) & =i[v(q, \gamma)-t],
\end{aligned}
$$

where $i>0$. This formulation is convenient since it makes $U, V$, and $U+V$ all quasi-linear. The scalar $i>0$ is the weight attached to the temptation utility and serves as the index of impulsiveness or the inverse of will power. As $i \rightarrow 0$, will power becomes infinite. The limit is a standard consumer with costless and complete self-control.

Additionally, we assume that $u$ and $v$ are $C^{2}$ and $C^{3}$, respectively, and satisfy the following assumptions.

Assumption 6. $v_{12}(q, \gamma)>0$.

Assumption 7. $v_{2}(q, \gamma)>0$ if $q>0$. 
Assumption 8. $v_{122}(q, \gamma) \leqslant 0$ and $v_{112}(q, \gamma) \geqslant 0 .^{7}$

Assumption 9. $f$ is $\log$-concave; i.e., $f^{\prime}(\gamma) / f(\gamma)$ is non-increasing in $\gamma$.

We also assume that $f$ and $C$ are $C^{1}$ and $C^{2}$, respectively, and the cost function also satisfies $C^{\prime}>0$ and $C^{\prime \prime}>0$.

The firm's problem is to maximize

$$
\int_{a}^{b}[u(q(\gamma))+i v(q(\gamma), \gamma)-w(\gamma)-(1+i) C(q(\gamma))] f(\gamma) d \gamma
$$

subject to that, for all $\gamma \in[a, b]$,

$$
\begin{aligned}
w^{\prime}(\gamma) & =i v_{2}(q(\gamma), \gamma), \\
w(\gamma) & \geqslant i[v(q(\gamma), \gamma)-u(q(\gamma))], \\
w(a) & =0, \\
q(\gamma) & \geqslant 0,
\end{aligned}
$$

$q$ is non-decreasing.

Here, $w(\gamma)$ denotes the ex post utility of type $\gamma$; i.e., $w(\gamma) \equiv u(q(\gamma))-t(\gamma)+i[v(q(\gamma), \gamma)-t(\gamma)]$. Constraint (7) is equivalent to $u-t \geqslant 0$. Since the values of $q(\cdot)$ at the points of discontinuity are immaterial, we only consider $q(\cdot)$ that is continuous from the left.

Following the standard approach in the nonlinear pricing literature, we first characterize the solution to the "relaxed" problem ignoring the monotonicity condition (8). The relaxed problem can be formulated as an optimal control problem in which $w$ is the state variable and $q$ is the control. The associated Hamiltonian is

$$
\begin{aligned}
H(w, q, \mu, \lambda, \gamma)= & {[u(q)+i v(q, \gamma)-w-(1+i) C(q)] f(\gamma) } \\
& +\mu i v_{2}(q, \gamma)+\lambda[i u(q)-i v(q, \gamma)+w] .
\end{aligned}
$$

As in the standard case, the log-concavity of $f$ (Assumption 9) guarantees the monotonicity of the quantity function $q(\cdot)$ characterized by the necessary conditions. We prove this as part of Proposition 7.

The ex ante IR condition in (7) places restrictions on the values that the state $w(\gamma)$ can take. As is well-known, when such state restrictions are present, a first-order approach (which uses the necessary conditions for the optimal control problem given by the Maximum Principle) may fail to isolate the optimal solution due to a constraint qualification (CQ) (see [22, p. 278, Note 4]). The CQ associated with the inequality constraint $i u-i v+w \geqslant 0$ states that the derivative of the constraint with respect to $q$ (i.e., $\left.i\left(u^{\prime}-v_{1}\right)\right)$ must not be equal to zero at all types at which the constraint binds. This implies that the CQ is violated if and only if $U=0$ (or equivalently $i u-i v+w=0$ ) for type $\gamma^{*}$. Hence, we consider two families of feasible schedules wherein the optimal schedule may lie: (1) those that violate the CQ, and (2) those that satisfy it.

\subsubsection{Case 1: optimal schedule violating $C Q$}

For a feasible tariff such that $U=0$ for $\gamma^{*}$, ex post and ex ante IR conditions imply that all types $\gamma<\gamma^{*}$ buy nothing and all types $\gamma>\gamma^{*}$ choose $x^{*}$. This implies that if the CQ is violated at an optimal schedule, the menu in Proposition 2 is optimal.

\footnotetext{
${ }^{7}$ This is A8 in Fudenberg and Tirole [12, p. 263].
} 


\subsubsection{Case 2: optimal schedule satisfying $C Q$}

Let $(q, w)$ be a pair that solves the relaxed problem and satisfies CQ. The necessary condition is that for all $\gamma \in[a, b]$,

$$
\begin{aligned}
& \quad\left[u^{\prime}(q(\gamma))+i v_{1}(q(\gamma), \gamma)-(1+i) C^{\prime}(q(\gamma))\right] f(\gamma)+\mu(\gamma) i v_{12}(q(\gamma), \gamma) \\
& \quad+\lambda(\gamma) i\left[u^{\prime}(q(\gamma))-v_{1}(q(\gamma), \gamma)\right]+\delta(\gamma)=0, \\
& \mu^{\prime}(\gamma)=f(\gamma)-\lambda(\gamma), \\
& \lambda(\gamma)[i u(q(\gamma)-i v(q(\gamma), \gamma)+w(\gamma)]=0, \quad \lambda(\gamma) \geqslant 0, \\
& \mu(b)=0, \\
& q(\gamma) \delta(\gamma)=0, \quad \delta(\gamma) \geqslant 0 .
\end{aligned}
$$

The solution to the relaxed problem can be characterized as follows.

Proposition 7. Suppose that a solution $(q, w)$ to the relaxed problem satisfies the CQ. Then $q$ is non-decreasing. Let

$$
\underline{\gamma} \equiv \max \{\gamma \in[a, b]: w(\gamma)=0\}
$$

and

$$
\bar{\gamma} \equiv \sup \{\gamma \in[a, b]: w(\gamma) / i>v(q(\gamma), \gamma)-u(q(\gamma))\}
$$

Then $\underline{\gamma}<\gamma^{*}<\bar{\gamma}$ and $q(\gamma)$ is given as follows:

1. For all $\gamma>\bar{\gamma}, q(\gamma)$ is constant. Let $\bar{q}$ denote the common quality level.

2. For all $\gamma \in[\underline{\gamma}, \bar{\gamma}]$,

$$
u^{\prime}(q(\gamma))+i v_{1}(q(\gamma), \gamma)-i v_{12}(q(\gamma), \gamma) \frac{\beta(\bar{\gamma})-F(\gamma)}{f(\gamma)}=(1+i) C^{\prime}(q(\gamma)),
$$

where $\beta(\bar{\gamma})$ is defined by

$$
\beta(\bar{\gamma}) \equiv F(\bar{\gamma})-\mu(\bar{\gamma})=F(\bar{\gamma})+(1-F(\bar{\gamma})) \frac{1+i}{i} \frac{C^{\prime}(\bar{q})-u^{\prime}(\bar{q})}{v_{1}(\bar{q}, \bar{\gamma})-u^{\prime}(\bar{q})} \in[F(\bar{\gamma}), 1] .
$$

If $\bar{\gamma}<b$, then $\beta(\bar{\gamma})>F(\bar{\gamma})$.

3. For all $\gamma<\underline{\gamma}, q(\gamma)=w(\gamma)=0$.

\subsubsection{The effect of temptation}

We now compare the optimal menu characterized above and the one in the absence of temptation, i.e., $V_{\gamma} \sim U$ for all $\gamma$. The comparison is useful since it shows how the presence of temptation affects the optimal menu. If $V_{\gamma} \sim U$ for all $\gamma$, temptation does not exist and the optimal menu is simply $M^{*}=\left\{x^{*}\right\}$. To compare this with the optimal menu in the presence of temptation, we divide the discussion based on whether the optimal menu satisfies the CQ. In what follows, let $M$ denote the optimal menu in the presence of temptation, i.e., the one that we characterized above.

Case 1: $C Q$ is violated. If $M$ does not satisfy the CQ, then, as shown above, $M=M^{*}$. However, consumers' choice within the menu is affected by their temptation. In the absence of temptation, 
consumers are indifferent between $x^{*}$ and $(0,0)$ and choose $x^{*}$. In the presence of temptation, all types $\gamma>\gamma^{*}$ prefer $x^{*}$ while all $\gamma<\gamma^{*}$ prefer $(0,0)$ in the strict sense. Nevertheless, all consumers get a zero ex ante utility whether or not there is temptation.

The firm's profits, on the other hand, depend on the presence of temptation. Since consumers $\gamma<\gamma^{*}$ with temptation choose $(0,0)$, the presence of temptation lowers the firm's profit by $\pi\left(x^{*}\right) F\left(\gamma^{*}\right)$.

Case 2: $C Q$ is satisfied. If the optimal menu in the presence of temptation satisfies the CQ, the effect of temptation is slightly different. First, the optimal menu with temptation gives all consumers a higher ex ante utility. Specifically, in the presence of temptation, $M$ satisfies $W_{\gamma}(M) \geqslant 0$ for all $\gamma$ and $W_{\gamma}(M)>0$ for all $\gamma \in(\underline{\gamma}, \bar{\gamma})$, while $M^{*}$ satisfies $W_{\gamma}\left(M^{*}\right)=0$ for all $\gamma$. Therefore, no consumer prefers $M^{*}$ to $M$. This is interesting since it implies that if consumers have temptation, they are better off if the firm is aware of the consumers' temptation. On the other hand, in the absence of temptation, we have $W_{\gamma}(M)>0=W_{\gamma}\left(M^{*}\right)$. Thus, even if temptation does not exist, consumers are better off if the firm believes it exists.

The firm's profits, on the other hand, are lower in the presence of temptation, as in the previous case. To see this, note that the optimal menu with temptation satisfies $U(x(\gamma)) \geqslant 0$ by ex ante IR. Proposition 7 implies $U(x(\gamma))>0$ for all $\gamma \in(\gamma, \bar{\gamma})$. Since $x^{*}$ is the solution of maximizing $\pi(x)$ subject to $U(x) \geqslant 0$, we have $\pi\left(x^{*}\right)>\int \pi(x(\gamma)) f(\gamma) d \gamma$.

Intuitively, there are two basic reasons for lower profits. First, since the menu must satisfy ex ante IR, the firm cannot take advantage of the consumers with upward temptation by charging high prices to high-quality goods. For those consumers, the menu such that $U(x(\gamma))<0$ can satisfy ex post IR but not ex ante IR. Second, temptation lowers some consumers' ex post willingness to pay and these consumers find $x^{*}$ too expensive to be acceptable. Therefore, to sell to these consumers, the menu has to offer them less profitable items.

Since the presence of temptation lowers the firm's profits and raises consumers' ex ante utility, it is natural to ask in what ways temptation affects "social welfare." Let us define social welfare as the sum of the expected profit of the firm and the expected ex ante utility of consumers: $\int\left[\pi(x(\gamma))+W_{\gamma}(M)\right] f(\gamma) d \gamma$. Since the ex ante utility is $U(x(\gamma))$ minus self-control costs, and $t(\gamma)$ is a transfer from consumers to the firm, the social welfare equals the expected value of $u(q(\gamma))-C(q(\gamma))$ minus self-control costs. Since $q(\gamma) \neq q^{*}$ and self-control costs are positive for a positive measure of consumers, social welfare is strictly lower in the presence of temptation.

To summarize the effect of temptation,

Proposition 8. A consumer's ex ante utility in the presence of temptation is at least as high as in the absence of temptation. If the optimal menu satisfies the $C Q$, some consumers get a strictly higher ex ante utility in the presence of temptation. The presence of temptation, on the other hand, lowers the firm's maximum profits, and the loss in the profit exceeds the gain in consumers' surplus.

\subsubsection{Comparison with the standard analysis}

We now compare the optimal menu and the solution to the standard nonlinear pricing problem with utility functions $U+V_{\gamma}$. The comparison is interesting since, if an economist were to analyze the current problem of the firm using the standard theory of nonlinear pricing, he would use $U+V_{\gamma}$ as the utility function of type- $\gamma$ consumers. Indeed, when our consumers choose within a menu, they maximize $U+V_{\gamma}$. We ask how the calculation of the optimal menu based on the standard theory differs from ours. As we show, there are a few non-trivial qualitative as well as quantitative differences. 
Throughout this section, the "standard problem" refers to the standard problem with utility functions $U+V_{\gamma}$. Let $M^{\mathrm{S}}=\operatorname{closure}\left\{\left(q^{\mathrm{S}}(\gamma), t^{\mathrm{S}}(\gamma)\right): \gamma \in[a, b]\right\}$ denote the optimal menu in the standard problem, which yields no surplus for $a$ and satisfies efficiency for $b$. Generally, we use superscript $\mathrm{S}$ to denote the variables for the standard problem. Let $M=\operatorname{closure}\{(q(\gamma), t(\gamma))$ : $\gamma \in[a, b]\}$ denote the optimal menu in our original problem.

Singleton menu: An obvious difference between $M$ and $M^{\mathrm{S}}$ is that $M$ may not satisfy the CQ, in which case $M$ contains a single non-zero choice. For $M^{\mathrm{S}}$, on the other hand, CQ is not an issue and therefore a menu with a single non-zero offer is never optimal.

The comparison is less trivial if the CQ is satisfied by $M$, since in this case $M$ is somewhat similar to $M^{\mathrm{S}}$. The necessary condition for $M^{\mathrm{S}}$ is

$$
\begin{aligned}
& u^{\prime}\left(q^{\mathrm{S}}(\gamma)\right)+i v_{1}\left(q^{\mathrm{S}}(\gamma), \gamma\right)-i v_{12}\left(q^{\mathrm{S}}(\gamma), \gamma\right) \frac{1-F(\gamma)}{f(\gamma)}=(1+i) C^{\prime}\left(q^{\mathrm{S}}(\gamma)\right) \text { for } \gamma \geqslant \underline{\gamma}^{\mathrm{S}}, \\
& q^{\mathrm{S}}(\gamma)=0 \text { for } \gamma<\underline{\gamma}^{\mathrm{S}} .
\end{aligned}
$$

The only difference between (17) and (15) is that $1-F(\gamma)$ in (17) becomes $\beta(\bar{\gamma})-F(\gamma)$ in (15). If $\bar{\gamma}=b$, then $\beta(\bar{\gamma})=1$ by (16) and hence the two equations coincide. Therefore, in what follows, we focus on the case with $\bar{\gamma}<b$. In this case, $\beta(\bar{\gamma})<1$ as we will show. Despite the apparent similarity between (17) and (15), a closer look reveals non-trivial differences between the two menus, as we now describe.

Inefficiency at the top: An important general property of optimal menus in the standard theory is that the offer chosen by the highest-type consumers is socially efficient. The so-called "efficiency at the top" property is lost in the current problem. Indeed, if $\bar{\gamma}<b$, Proposition 7 says $\beta(\bar{\gamma})>F(\bar{\gamma})$. Therefore, (15) for $\bar{\gamma}$ implies

$$
\frac{u^{\prime}(\bar{q})+i v_{1}(\bar{q}, \bar{\gamma})}{1+i}>C^{\prime}(\bar{q})
$$

The left-hand side is the marginal value of quality for the utility function $U+V_{\bar{\gamma}}$. Since the marginal value is increasing in $\gamma$, the inequality holds for all $\gamma \geqslant \bar{\gamma}$, including the highest type. The inequality shows that there is room for a Pareto improvement between the firm and the consumer if the consumer has chosen the menu. Specifically, there exists an offer $y$ that the consumer prefers to $x(\gamma)$ in the post-entry stage and generates higher profits. Formally, there is an offer $y$ such that $U(y)+V_{\gamma}(y)>U(x(\gamma))+V_{\gamma}(x(\gamma))$ and $\pi(y)>\pi(x(\gamma))$. However, the firm does not want to include $y$ in the menu since $y$ is not desirable for the consumer's commitment utility, and if the consumer knows that $y$ is available, he will stay away from the menu.

By substituting (18) into (16), we obtain $\beta(\bar{\gamma})<1$, as mentioned earlier.

Bunching at the top: Another non-standard feature of the optimal menu $M$ is the possibility that consumers of highest types are bunched together. Proposition 7 shows that if $\bar{\gamma}<b$, all consumers $\gamma>\bar{\gamma}$ choose the same offer from $M$. It appears, therefore, as if there were a price regulation that sets a price ceiling at $t(b)$. Indeed, the optimal menu $M$ resembles the menu in Besanko et al. [3], who study nonlinear pricing when there is a price ceiling. In their problem, however, the price ceiling is exogenous. In our problem, the constraint is $U(x(\gamma)) \geqslant 0$ coming from ex ante IR.

We now describe the quantitative differences between $M$ and $M^{\mathrm{S}}$.

Consumers who buy: We first compare the lowest type of consumers who participate. Eq. (15) can be written as $B_{q}(q(\gamma), \gamma)=0$, where $B(q, \gamma)$ is defined by

$$
B(q, \gamma) \equiv u(q)+i v(q, \gamma)-i v_{2}(q, \gamma) \frac{\beta(\bar{\gamma})-F(\gamma)}{f(\gamma)}-(1+i) C(q)
$$


Appendix A.6 shows $B_{q q}<0$ and $B_{q \gamma}>0$ (see the proof of Claim 8). With the definition, (15) says $B_{q}(q(\underline{\gamma}), \underline{\gamma})=0$. On the other hand, (17) says

$$
B_{q}\left(q^{\mathrm{S}}\left(\underline{\gamma}^{\mathrm{S}}\right), \underline{\gamma}^{\mathrm{S}}\right)=i v_{12}\left(q^{\mathrm{S}}\left(\underline{\gamma}^{\mathrm{S}}\right), \underline{\gamma}^{\mathrm{S}}\right) \frac{1-\beta(\bar{\gamma})}{f\left(\underline{\gamma}^{\mathrm{S}}\right)}>0=B_{q}(q(\underline{\gamma}), \underline{\gamma}) \text {. }
$$

The inequality follows from $\beta(\bar{\gamma})<1$ and $v_{12}>0$. Suppose, for the moment, that $\underline{\gamma}, \underline{\gamma}^{\mathrm{S}}>a$. Then by the left-continuity, $q(\underline{\gamma})=q^{\mathrm{S}}\left(\underline{\gamma}^{\mathrm{S}}\right)=0$. Then, since $B_{q \gamma}>0$, the inequality in (20) yields $\underline{\gamma}^{\mathrm{S}}>\underline{\gamma}$. That is, the standard analysis predicts that the lowest type served is higher. As the following proposition shows, $\underline{\gamma}^{\mathrm{S}}=\underline{\gamma}$ is possible but only if $\underline{\gamma}^{\mathrm{S}}=\underline{\gamma}=a$.

Proposition 9. Either $\underline{\gamma}^{\mathrm{S}}>\underline{\gamma}$ or $\underline{\gamma}^{\mathrm{S}}=\underline{\gamma}=a$.

Qualities chosen by consumers: We now see how the quality levels chosen by consumers differ for $M$ and $M^{\mathrm{S}}$. Eqs. (17) and (19) imply that for all $\gamma \in\left[\underline{\gamma}^{\mathrm{S}}, \bar{\gamma}\right]$,

$$
B_{q}\left(q^{\mathrm{S}}(\gamma), \gamma\right)=i v_{12}\left(q^{\mathrm{S}}(\gamma), \gamma\right) \frac{1-\beta(\bar{\gamma})}{f(\gamma)}>0=B_{q}(q(\gamma), \gamma)
$$

The inequality implies $q^{\mathrm{S}}(\gamma)<q(\gamma)$, which holds for all $\gamma \in(\underline{\gamma}, \bar{\gamma}]$. For these consumers, the standard analysis predicts lower quality levels.

To obtain intuition, recall that in the standard nonlinear pricing problem, the firm deteriorates the quality sold to low-type consumers, and excludes some of them, in order to increase the surplus extracted from the high-type ones. With self-control preferences, the ex ante IR condition imposes an effective bound on the feasibility of price discrimination against higher type consumers. As a result, the firm has less incentives to deteriorate the quality sold to lower types, or exclude them, than it does in the standard problem.

To extend the comparison to types $\gamma>\bar{\gamma}$, first consider the highest type, $b$. While inequality (18) holds for $\gamma=b$, (17) yields $u^{\prime}\left(q^{\mathrm{S}}(b)\right)+i v_{1}\left(q^{\mathrm{S}}(b)\right)=(1+i) C^{\prime}\left(q^{\mathrm{S}}(b)\right)$, i.e., there is "efficiency at the top" in the standard problem. Thus, $q^{\mathrm{S}}(b)>\bar{q}$. It follows that there exists a threshold type $\hat{\gamma} \in(\bar{\gamma}, b)$ such that

$$
\begin{array}{ll}
q^{\mathrm{S}}(\gamma)>\bar{q} & \text { if } \gamma>\hat{\gamma}, \\
q^{\mathrm{S}}(\gamma)<\bar{q} & \text { if } \bar{\gamma}<\gamma<\hat{\gamma} .
\end{array}
$$

Fig. 2 summarizes the comparison between $q(\gamma)$ and $q^{\mathrm{S}}(\gamma)$.

Prices: We can also compare the prices. For each quality level $q$, let $P(q)$ denote the price for the quality level in menu $M$. Formally,

$$
P(q) \equiv \begin{array}{ll}
t(\gamma) & \text { if there exists } \gamma \in[a, b] \text { such that } q(\gamma)=q \\
+\infty & \text { otherwise. }
\end{array}
$$

This is well-defined since $t(\gamma)$ is identical for all $\gamma$ that have the same value of $q(\gamma)$. For $M^{\mathrm{S}}$, we can define $P^{\mathrm{S}}(q)$ similarly. The following result compares $P(q)$ and $P^{\mathrm{S}}(q)$.

Proposition 10. The optimal menu charges a lower price than the optimal menu in the standard problem for any good that is offered in both menus: $P(q)<P^{\mathrm{S}}(q)$ for any $q>0$ such that $P(q)$ and $P^{\mathrm{S}}(q)$ are both finite. 


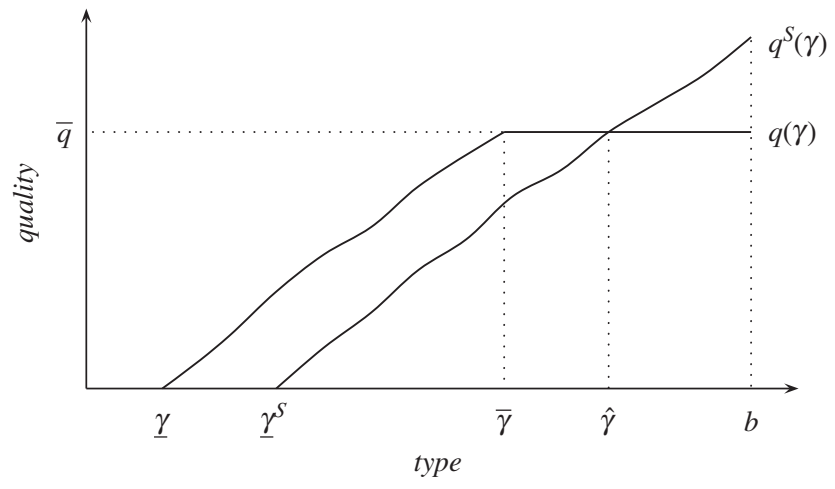

Fig. 2. The quality level chosen by each type.

Proof. Let $q>0$ be as in the proposition. Since $P(q)$ is finite, there exist $t \in \mathbb{R}_{+}$and $\gamma \in[a, b]$ such that $(q, t)=x(\gamma)$. Similarly, there exist $t^{\mathrm{S}} \in \mathbb{R}_{+}$and $\gamma^{\mathrm{S}} \in[a, b]$ such that $\left(q, t^{\mathrm{S}}\right)=x\left(\gamma^{\mathrm{S}}\right)$.

Here, we consider only the case where $\gamma<\gamma \leqslant \bar{\gamma}$. The other cases are considered in the Appendix. By the inequalities,

$$
\begin{aligned}
U(q, t)+V_{\gamma}(q, t)=w(\gamma) & =\int_{a}^{\gamma} i v_{2}(q(y), y) d y \\
& >\int_{a}^{\gamma} i v_{2}\left(q^{\mathrm{S}}(y), y\right) d y=w^{\mathrm{S}}(\gamma) \geqslant U\left(q, t^{\mathrm{S}}\right)+V_{\gamma}\left(q, t^{\mathrm{S}}\right) .
\end{aligned}
$$

The first inequality follows from that $q(y)>q^{\mathrm{S}}(y)$ for all $y \in(\gamma, \gamma]$, and the second one follows from ex post IC. The inequalities imply $t<t^{\mathrm{S}}$.

Profits: Let $\Pi$ and $\Pi^{\mathrm{S}}$ denote the expected profit from $M$ and $M^{\mathrm{S}}$, respectively. Since the standard problem has fewer constraints than our problem, $\Pi^{\mathrm{S}} \geqslant \Pi{ }^{8}$ If $\bar{\gamma}<b$ then $\Pi^{\mathrm{S}}>\Pi$.

Welfare: An advantage of Gul and Pesendorfer's utility formulation is that it has a clear notion of ex ante welfare. We take advantage of it and compare $W_{\gamma}(M)$ and $W_{\gamma}\left(M^{\mathrm{S}}\right)$. To facilitate the comparison, we assume that $V_{\gamma}$ is defined for all $\gamma \in \mathbb{R}$ so that, for any $\gamma \in[a, b]$, there exists $\theta_{\gamma} \in \mathbb{R}$ such that $U+V_{\theta_{\gamma}}$ has the same ordinal preferences as $V_{\gamma}$. For the current quasi-linear formulation, this means

$$
\frac{u(q)+i v\left(q, \theta_{\gamma}\right)}{1+i}=v(q, \gamma) \text { for all } q
$$

Thus, the requirement is that each $v(\cdot, \gamma)$ can be written as the weighted average of $u(\cdot)$ and $v\left(\cdot, \theta_{\gamma}\right)$ for some $\theta_{\gamma}$. If this is the case, then $\theta_{\gamma}>\gamma$ if $\gamma>\gamma^{*}$ and $\theta_{\gamma}<\gamma$ if $\gamma<\gamma^{*}$. For example, this assumption is satisfied in the linear environment where $v(q, \gamma)=\gamma q, u(q)=q$, and $\gamma^{*}=1$. It is possible that $\theta_{\gamma}$ is outside the interval $[a, b]$, but this is not a problem, as we discuss later. We begin, however, with the case where $\theta_{\gamma} \in[a, b]$.

${ }^{8}$ A proof goes as follows. Proposition 1 implies that in our original problem, there exists a feasible schedule $(\hat{M}, \hat{x}, \Gamma)$ such that the expected profit equals $\Pi$. Since all types enter, ex post IR and IC are satisfied by all types, which implies that $(\hat{M}, \hat{x})$ is a feasible schedule in the standard problem. The expected profit of the schedule, $\Pi$, therefore, gives a lower bound of $\Pi^{\mathrm{S}}$. 
Consider a type $\gamma$ such that $\theta_{\gamma} \in[a, b]$. The consumer's ex ante utility from $M$ is then

$$
\begin{aligned}
W_{\gamma}(M)= & {[u(q(\gamma))+i v(q(\gamma), \gamma)-(1+i) t(\gamma)] } \\
& -\frac{i}{1+i}\left[u\left(q\left(\theta_{\gamma}\right)\right)+i v\left(q\left(\theta_{\gamma}\right), \theta_{\gamma}\right)-(1+i) t\left(\theta_{\gamma}\right)\right] \\
= & w(\gamma)-\frac{i}{1+i} w\left(\theta_{\gamma}\right) \\
= & \int_{a}^{\gamma} i v_{2}(q(y), y) d y-\frac{i}{1+i} \int_{a}^{\theta_{\gamma}} i v_{2}(q(y), y) d y \\
= & \frac{1}{1+i} \int_{a}^{\gamma} i v_{2}(q(y), y) d y+\frac{i}{1+i} \int_{\theta_{\gamma}}^{\gamma} i v_{2}(q(y), y) d y .
\end{aligned}
$$

We can also compute $W_{\gamma}\left(M^{\mathrm{S}}\right)$ by replacing $q(\gamma)$ with $q^{\mathrm{S}}(\gamma)$ in (22). Now, consider any type $\gamma<\gamma^{*}$. As stated above, $\theta_{\gamma}<\gamma$ and $q^{\mathrm{S}}(y) \leqslant q(y)$ for all $y<\gamma$. Therefore, replacing $q(y)$ with $q^{\mathrm{S}}(y)$ lowers the value of (22) weakly. If $\gamma>\underline{\gamma}$, then $q^{\mathrm{S}}(y)<q(y)$ for all $y \in(\underline{\gamma}, \gamma)$ and therefore the value of (22) decreases strictly. This gives a partial proof of the following.

Proposition 11. For all consumers with downward temptation, the optimal menu gives a higher ex ante utility than the optimal menu in the standard problem, except for the consumers who buy nothing under both menus: $W_{\gamma}(M)>W_{\gamma}\left(M^{\mathrm{S}}\right)$ for all $\gamma$ such that $\underline{\gamma}<\gamma<\gamma^{*}$, and $W_{\gamma}(M)=$ $W_{\gamma}\left(M^{\mathrm{S}}\right)=0$ for all $\gamma<\underline{\gamma}$.

Proof. The above proof is not complete since we assumed $\theta_{\gamma} \in[a, b]$. To complete the proof, therefore, we need to consider types $\gamma$ such that $\gamma<\gamma^{*}$ and $\theta_{\gamma}<a$. For these types, $V_{\gamma}$ has flatter indifference curves than $U+V_{a}$. This implies $\max _{x \in M} V_{\gamma}(x)=0=\max _{x \in M^{\mathrm{s}}} V_{\gamma}(x)$ since ex post IR binds for type $a$ in both menus. Therefore,

$$
W_{\gamma}(M)=w(\gamma)=\int_{a}^{\gamma} i v_{2}(q(y), y) d y \geqslant \int_{a}^{\gamma} i v_{2}\left(q^{\mathrm{S}}(y), y\right) d y=w^{\mathrm{S}}(\gamma)=W_{\gamma}\left(M^{\mathrm{S}}\right) .
$$

As before, the inequality is strict if $\gamma>\underline{\gamma}$.

For consumers with upward temptation, the comparison between $W_{\gamma}(M)$ and $W_{\gamma}\left(M^{\mathrm{S}}\right)$ is generally ambiguous. However, we can make a clear statement for sufficiently high types.

Proposition 12. For a high end of consumers with upward temptation, the optimal menu yields a higher ex ante utility than the optimal menu in the standard problem: for all $\gamma \geqslant \hat{\gamma}, W_{\gamma}(M)>$ $W_{\gamma}\left(M^{\mathrm{S}}\right)$.

Proof. Since $\hat{\gamma}>\bar{\gamma}$, all consumers $\gamma \geqslant \hat{\gamma}$ choose the same offer $\bar{x}=(\bar{q}, \bar{t})$ from $M$. By the definition of $\bar{\gamma}$ in (14), we have $w(\gamma) / i=v(\bar{q}, \gamma)-u(\bar{q})$, which implies $U(\bar{x})=0$. Since $\bar{q}$ is the maximum quality in $M$, the offer $\bar{x}$ also maximizes $V_{\gamma}$ in the menu. Therefore, $W_{\gamma}(M)=0$.

What remains to be shown is $W_{\gamma}\left(M^{\mathrm{S}}\right)<0$. We start with type $\hat{\gamma}$. By definition, $q^{\mathrm{S}}(\hat{\gamma})=\bar{q}$. Proposition 10 implies $t^{\mathrm{S}}(\hat{\gamma})>\bar{t}$. Thus, $W_{\hat{\gamma}}\left(M^{\mathrm{S}}\right) \leqslant U\left(x^{\mathrm{S}}(\hat{\gamma})\right)<U(\bar{x})=0$.

Now, consider any type $\gamma>\hat{\gamma}$. By ex post IC, $x^{\mathrm{S}}(\gamma) \geqslant x^{\mathrm{S}}(\hat{\gamma})$, and $x^{\mathrm{S}}(\gamma)$ is not better than $x^{\mathrm{S}}(\hat{\gamma})$ for $U+V_{\hat{\gamma}}$. Since $U+V_{\hat{\gamma}}$ has steeper indifference curves than $U$ and $U\left(x^{\mathrm{S}}(\hat{\gamma})\right)<0$, it follows that $U\left(x^{\mathrm{S}}(\gamma)\right)<0$. Therefore, $W_{\gamma}\left(M^{\mathrm{S}}\right) \leqslant U\left(x^{\mathrm{S}}(\gamma)\right)<0$. 
Here is the intuition behind the comparisons between $W_{\gamma}(M)$ and $W_{\gamma}\left(M^{\mathrm{S}}\right)$. The main difference between $M$ and $M^{\mathrm{S}}$ is that $M$ charges lower prices for the goods it offers, but its product selection is limited toward the high end. Obviously, having lower prices has a positive effect on consumers' welfare. But there is a negative effect as well since tempting products are also offered at lower prices, which may induce a stronger temptation and make self-control more costly. The total effect on welfare is therefore ambiguous generally.

For consumers with downward temptation, however, the positive effect dominates the negative one. The reason is that tempting choices for consumers with downward temptation are low-quality goods, for which the price reduction is relatively small. Indeed, $P^{\prime}(q)$ is equal to the marginal value of quality for $U+V_{\gamma}$ where $\gamma$ is the one such that $q(\gamma)=q$. Thus, Fig. 2 implies $P^{\prime}(q)<P^{S \prime}(q)$ for all $q \leqslant \bar{q}$. The price difference is larger for higher quality goods.

The reason why we also obtain a clear welfare comparison for high-valuation consumers is that the consumers who choose the highest-quality product do not see anything more tempting. As a result, those consumers incur no self-control cost and fully enjoy the benefit of having low prices. In contrast, the welfare comparison is ambiguous for consumers $\gamma \in\left(\gamma^{*}, \hat{\gamma}\right)$ since for these consumers the optimal compromise between $U$ and $V$ is located in the middle of $M$ and the presence of higher-quality products inflicts self-control costs. For these consumers, it is theoretically possible that the loss from having larger self-control costs exceeds the gain in the commitment utility from having lower prices.

\section{Conclusion}

We have considered consumers for whom self-control is costly and characterized a monopolist's optimal nonlinear pricing schedule. Costly self-control makes the no-exit constraint (ex post IR) different from the entry constraint (ex ante IR), and the entry constraint is what makes the optimal menu qualitatively different from the one in the standard theory. In the first part of the paper, we saw that there exists an asymmetry between upward and downward temptation. If all consumers have upward temptation, the firm offers a small menu and extracts the entire surplus. If all consumers have downward temptation, the optimal menu looks like one in the standard model. The second half of the paper analyzed the case where both types of consumers exist. In the mixed case, the optimal menu is either the same as in the absence of temptation or resembles the one in the standard model with a price ceiling. In either case, the entry constraint binds for high-valuation consumers and their choice exhibits bunching and inefficiency.

One message of our analysis is that the existence of temptation does not imply an advantage to firms. The comparison between the worlds with and without temptation showed that the firm does not gain from the presence of temptation. Firms are not better off because the entry constraint prevents them from selling tempting products for high prices. The entry constraint must be satisfied because consumers foresee their temptation. In our model, therefore, the foresight of consumers is sufficient to offset the firm's advantage. ${ }^{9}$

Our simplifying assumptions make the conclusions less general. In particular, we assumed that consumers differ only in their temptation preferences. Allowing both utility functions to depend on the type makes it difficult to apply the existing technique of mechanism design. Esteban and Miyagawa [9] deal with the issue by studying a two-type model. Obtaining a general solution

\footnotetext{
${ }^{9}$ Of course, in reality, firms may be able to trick consumers occasionally, by hiding product attributes or advertising deceptively. However, this can also happen with standard preferences and the presence of temptation per se does not imply an advantage to firms.
} 
will be challenging. Another important assumption in the present study is that the firm is not allowed to offer more than one menu. Since consumers have a preference for small menus, the firm may have an incentive to offer multiple small menus, which is the main topic of Esteban and Miyagawa [9]. In a separate paper, Esteban and Miyagawa [10] study how the optimal pricing is affected by the presence of rival firms.

\section{Acknowledgments}

We thank the editor, Alessandro Lizzeri, and an anonymous referee for helpful comments, which greatly improved the paper. We thank Eric Bond, Kalyan Chatterjee, Matthew Jackson, and Larry Samuelson for helpful comments and discussion. We also thank the participants of the SED 2002 Conference at New York University, the Henry Luce 2003 Conference at the Pennsylvania State University, the Clarence W. Tow 2003 Conference at the University of Iowa, and seminars at Concordia University and Tokyo Institute of Technology. An earlier draft of this paper was titled "Optimal Pricing of Temptation Goods."

\section{Appendix A}

\section{A.1. Proof of Proposition 1}

Let $(M, x, E)$ be a feasible schedule. Define

$$
\begin{aligned}
& M_{1} \equiv\left\{y \in \mathbb{R}_{+}^{2}: y=x(\gamma) \text { for some } \gamma \in \Gamma\right\}, \\
& M_{2} \equiv\left\{y \in \mathbb{R}_{+}^{2}: \pi(y) \geqslant 0\right\}, \\
& M_{3} \equiv \text { closure }\left[\left(M_{1} \cap M_{2}\right) \cup\{(0,0)\}\right] .
\end{aligned}
$$

Since $M$ is compact, so is $M_{3}$. For all types $\gamma \in E$ such that $x(\gamma) \in M_{3}$, define $\hat{x}(\gamma) \equiv x(\gamma)$. For all other types, let $\hat{x}(\gamma) \in M_{3}$ be an offer that maximizes $U+V_{\gamma}$. Then let

$$
M_{4} \equiv \text { closure }\left\{y \in \mathbb{R}_{+}^{2}: y=\hat{x}(\gamma) \text { for some } \gamma \in \Gamma\right\} .
$$

We claim that a schedule given by $\left(M_{4}, \hat{x}, \Gamma\right)$ is a desired schedule. Property 2 is satisfied since any $y \in M_{1}$ satisfies $U(y) \geqslant 0$ by Corollary 1 . Properties 1,3 , and 4 are satisfied obviously. The schedule is at least as profitable as the initial one since for all types such that $\hat{x}(\gamma) \neq x(\gamma), x(\gamma)$ yields non-positive profits while $\hat{x}(\gamma)$ yields non-negative profits.

\section{A.2. Proof of Corollary 3}

"If" part. Suppose that $(M, x)$ is an optimal schedule for the standard problem with utility functions $U+V_{\gamma}$. Since the ex post incentive constraints are satisfied for all types, $(M, x, \Gamma)$ is a feasible schedule for the original problem. To show that it is also optimal, suppose that a feasible schedule $\left(M^{\prime}, x^{\prime}, E^{\prime}\right)$ yields higher profits. By Proposition 1 , we can assume $E^{\prime}=\Gamma$. But then, $\left(M^{\prime}, x^{\prime}\right)$ is feasible for the standard problem yielding higher profits than $(M, x)$, a contradiction.

"Only if" part. Let $(M, x, E)$ be an optimal schedule for the original problem. First, observe that Proposition 3 does not imply $E=\Gamma$ since some consumers may not enter the store if $W_{\gamma}(M)=0$. But, we first consider the case where $E=\Gamma$. The ex post incentive constraints are therefore satisfied for all types and hence $(M, x)$ is a feasible schedule for the standard problem. The schedule is also optimal since if it is dominated by another schedule $\left(M^{\prime}, x^{\prime}\right)$ then 
$\left(M^{\prime}, x^{\prime}, \Gamma\right)$ is a feasible schedule for the original problem yielding larger profits than $(M, x, \Gamma)$, a contradiction.

Now, consider the case where $E \subseteq \Gamma$. We show that we can actually set $E=\Gamma$, i.e., $(M, x, \Gamma)$ is also an optimal schedule for the original problem, which then enables us to use the argument in the previous paragraph. All we need to show is that the assignment function $x$ satisfies ex post IC for all $\gamma \notin E$. So, let $\gamma \notin E$. By Proposition 3, $W_{\gamma}(M)=0$. The proof of the proposition shows that, in (6), the inequality holds with equality and $U(y)=0$, where $y \in \operatorname{argmax}_{z \in M} V_{\gamma}(z)$. Since $\gamma \leqslant \gamma^{*}, U(y)=0$ implies $V_{\gamma}(y) \leqslant 0$. Since $y$ also maximizes $U+V_{\gamma}$ and $(0,0)$ is available, we obtain $\max _{z \in M} U(z)+V_{\gamma}(z)=0$. Since $x(\gamma)=(0,0), x$ satisfies ex post IC for $\gamma$.

\section{A.3. Proof of Proposition 4}

Let $(M, x, \Gamma)$ be a regular optimal schedule. Since $M$ may not be a connected set, we first fill the holes.

For all $(x, \gamma) \in \mathbb{R}_{+}^{2} \times \Gamma$, let $\xi(x, \gamma)$ be defined by

$$
\xi(x, \gamma) \equiv U(x)+V_{\gamma}(x) .
$$

This is simply the ex post utility of an offer $x$ for type $\gamma$. Let

$$
L_{\gamma} \equiv\left\{x \in \mathbb{R}_{+}^{2}: \xi(x(\gamma), \gamma) \geqslant \xi(x, \gamma)\right\} \quad \text { and } \quad L \equiv \cap\left\{L_{\gamma}: \gamma \in[a, b]\right\} .
$$

Since $L_{\gamma}$ is closed, so is $L$. For all $q \in[q(a), q(b)]$, there exists $t \geqslant 0$ such that $(q, t) \in L$ (e.g., $t(b))$. Since $L$ is a closed subset of $\mathbb{R}_{+}^{2}$, we can define $T:[q(a), q(b)] \rightarrow \mathbb{R}_{+}$by

$$
T(q) \equiv \min \left\{t \in \mathbb{R}_{+}:(q, t) \in L\right\} .
$$

Then for all $q$,

$$
\xi(x(\gamma), \gamma) \geqslant \xi(q, T(q), \gamma) \text { for all } \gamma \text {. }
$$

The inequality implies that the ex post IC of $x(\cdot)$ is preserved in the enlarged menu $\{(q, T(q))$ : $q \in[q(a), q(b)]\}$. The following lemma shows that the inequality holds with equality for some $\gamma$.

Lemma 3. For all $q \in[q(a), q(b)]$, there exists a type $\gamma=\hat{\gamma}(q)$ such that $\xi(x(\gamma), \gamma)=$ $\xi(q, T(q), \gamma)$.

Proof. Suppose not. That is, there exists $\hat{q} \in[q(a), q(b)]$ such that

$$
\xi(x(\gamma), \gamma)>\xi(\hat{q}, T(\hat{q}), \gamma) \text { for all } \gamma \text {. }
$$

The inequality implies $q(\gamma) \neq \hat{q}$ for all $\gamma$; for if $q(\gamma)=\hat{q}$ then $T(\hat{q})=t(\gamma)$. Since $q(\gamma)$ is non-decreasing, there exists a unique type $\hat{\gamma}$ at which $q(\cdot)$ jumps over $\hat{q}$. That is, $q(\gamma)<\hat{q}<q\left(\gamma^{\prime}\right)$ for all $\gamma, \gamma^{\prime}$ such that $\gamma<\hat{\gamma}<\gamma^{\prime}$. Without loss of generality, assume $q(\hat{\gamma})>\hat{q}$. The case in which $q(\hat{\gamma})<\hat{q}$ can be dealt with symmetrically. By (23), there exists $\hat{t}<T(\hat{q})$ such that $\xi(x(\hat{\gamma}), \hat{\gamma})>\xi(\hat{q}, \hat{t}, \hat{\gamma})$. By ex post IC and single-crossing,

$$
\xi(x(\gamma), \gamma) \geqslant \xi(x(\hat{\gamma}), \gamma)>\xi(\hat{q}, \hat{t}, \gamma) \quad \text { for all } \gamma \geqslant \hat{\gamma} \text {. }
$$


Now, consider any strictly increasing sequence $\left\{\gamma_{k}\right\}$ converging to $\hat{\gamma}$. Since $x(\gamma)$ is non-decreasing, $x\left(\gamma_{k}\right)$ converges to some point $\tilde{x}$. By ex post IC,

$$
\begin{gathered}
\xi\left(x\left(\gamma_{k}\right), \gamma_{k}\right) \geqslant \xi\left(x(\hat{\gamma}), \gamma_{k}\right), \\
\xi\left(x\left(\gamma_{k}\right), \hat{\gamma}\right) \leqslant \xi(x(\hat{\gamma}), \hat{\gamma}) .
\end{gathered}
$$

Since $\xi$ is continuous, taking the limit gives $\xi(\tilde{x}, \hat{\gamma})=\xi(x(\hat{\gamma}), \hat{\gamma})$. Since $q\left(\gamma_{k}\right)<\hat{q}$ for all $k$, we have $\tilde{q} \leqslant \hat{q}$. By ex post IC with single-crossing,

$$
\xi(x(\gamma), \gamma) \geqslant \xi(\tilde{x}, \gamma) \geqslant \xi(\hat{q}, \hat{t}, \gamma) \text { for all } \gamma \leqslant \hat{\gamma} .
$$

This and (24) are in contradiction with the minimality of $T(\hat{q})$ since $\hat{t}<T(\hat{q})$.

Lemma 4. Function $T$ is strictly increasing.

Proof. Let $q<q^{\prime}$ and $\gamma=\hat{\gamma}(q)$. Then since $\xi(q, T(q), \gamma) \geqslant \xi\left(q^{\prime}, T\left(q^{\prime}\right), \gamma\right)$ and preferences are strictly monotone, $T\left(q^{\prime}\right)>T(q)$.

\section{Lemma 5. Function $T$ is continuous.}

Proof. Take any $q \in[q(a), q(b)]$. Let $\bar{t}$ and $\underline{t}$ be the right and left limit of $T\left(q^{\prime}\right)$ as $q^{\prime} \rightarrow q$, respectively. Since $T$ is monotonic, $\underline{t} \leqslant T(q) \leqslant \bar{t}$. Let $\gamma=\hat{\gamma}(q)$. Take a sequence $\left\{q_{k}\right\}$ that converges to $q$ from the left. Then $\xi(q, T(q), \gamma) \geqslant \xi\left(q_{k}, T\left(q_{k}\right), \gamma\right)$. Taking the limit yields $\xi(q, T(q), \gamma) \geqslant$ $\xi(q, \underline{t}, \gamma)$. This implies $T(q)=\underline{t}$.

Now, take a different sequence $\left\{q_{k}\right\}$ that converges to $q$ from the right. For each $k$, let $\gamma_{k}=$ $\hat{\gamma}\left(q_{k}\right)$. Then $\xi\left(q_{k}, T\left(q_{k}\right), \gamma_{k}\right) \geqslant \xi\left(q, \underline{t}, \gamma_{k}\right)$. Since $\gamma_{k} \in[a, b]$, there exists a subsequence of $\left\{\gamma_{k}\right\}$ converging to some $\tilde{\gamma} \in[a, b]$. Since $\xi$ is continuous, taking the limit yields $\xi(q, \bar{t}, \tilde{\gamma}) \geqslant \xi(q, \underline{t}, \tilde{\gamma})$, which implies $\bar{t}=\underline{t}$.

To prove the proposition by way of contradiction, suppose $\xi(x(a), a)>0$. We divide the argument into a few steps.

Step 1 (Preliminaries): We first consider the case when

$$
\xi(q, T(q), a) \text { is constant over all } q \in[q(a), q(b)] .
$$

That is, all offers in the enlarged menu lie on a single indifference curve of type $a$. By ex post IC, $q(\gamma)=q(b)$ for all $\gamma>a$. That is, $M$ consists of two items. Let

$$
\gamma^{\prime} \in \underset{\gamma \in\{a, b\}}{\operatorname{argmax}} \pi(x(\gamma))
$$

Then for a sufficiently small $\delta>0$, a singleton menu $\left\{\left(q\left(\gamma^{\prime}\right), t\left(\gamma^{\prime}\right)+\delta\right)\right\}$ satisfies both conditions of IR and generates more profits than $(M, x, \Gamma)$, in contradiction with the optimality of $(M, x, \Gamma)$.

Therefore, in what follows, we assume that there exists $k \in(q(a), q(b))$ such that

$$
\xi(x(a), a)>\xi(k, T(k), a) .
$$

Since $\xi$ and $T$ are continuous, we can choose $k>q(a)$ sufficiently small to satisfy

$$
\xi(q, T(q), a)>0 \quad \text { for all } q \in[q(a), k] .
$$


Let $\rho:[q(a), q(b)] \rightarrow \mathbb{R}$ be defined by

$$
\rho(q) \equiv T(q)-C(q) .
$$

Since this function is continuous, there exists a quantity level $q^{m} \in[q(a), k]$ that maximizes $\rho$ over $[q(a), k]$. By $(26)$, there exists a small number $\varepsilon>0$ such that

$$
\xi\left(q^{m}, T\left(q^{m}\right)+\varepsilon, a\right)>0 .
$$

Step 2 (Construction of a schedule): We now construct a schedule that generates more profits than $(M, x, \Gamma)$. We basically consider the upper envelope of $T$ and the isoprofit curve that passes through $\left(q^{m}, \hat{t}\left(q^{m}\right)+\varepsilon\right)$. Formally, let $\tau:[q(a), q(b)] \rightarrow \mathbb{R}$ be defined by

$$
\tau(q) \equiv \max \left\{T(q), C(q)+\rho\left(q^{m}\right)+\varepsilon\right\} .
$$

We remove the part of the upper envelope where $U$ is negative. Formally, let

$$
D \equiv\{q \in[q(a), q(b)]: U(q, \tau(q)) \geqslant 0\} .
$$

Then $D$ is compact and contains $q^{m}$. The menu we consider is

$$
M^{\prime} \equiv\{(q, \tau(q)): q \in D\} .
$$

Let $x^{\prime}:[a, b] \rightarrow M^{\prime}$ be an assignment function such that, for all $\gamma \in[a, b]$,

$$
x^{\prime}(\gamma) \in \underset{x \in M^{\prime}}{\operatorname{argmax}} \xi(x, \gamma),
$$

with the tie-breaking rule that if $x(\gamma)$ is a solution of (29) then $x^{\prime}(\gamma)=x(\gamma)$.

Step 3 (Feasibility): We show that $\left(M^{\prime}, x^{\prime}, \Gamma\right)$ satisfies all the incentive constraints. The ex post IC condition follows from (29). The ex ante IR condition holds for all types because of the definition of $D$. The ex post IR condition also holds for all types since (27) implies that type $a$ obtains a positive ex post utility.

Step 4: We show that in terms of the ex post utilities, all types are weakly worse off in $M^{\prime}$ than $M$. Indeed, for all $\gamma \in[a, b]$,

$$
w(M, \gamma) \geqslant \xi\left(q^{\prime}(\gamma), T\left(q^{\prime}(\gamma)\right), \gamma\right) \geqslant \xi\left(q^{\prime}(\gamma), \tau\left(q^{\prime}(\gamma)\right), \gamma\right)=w\left(M^{\prime}, \gamma\right) .
$$

Step 5: We prove that $\left(M^{\prime}, x^{\prime}, \Gamma\right)$ is at least as profitable as $(M, x, \Gamma)$. We actually prove a stronger statement: each type generates weakly more profits in $\left(M^{\prime}, x^{\prime}, \Gamma\right)$ than in $(M, x, \Gamma)$, i.e.,

$$
\tau\left(q^{\prime}(\gamma)\right)-C\left(q^{\prime}(\gamma)\right) \geqslant \rho(q(\gamma)) \text { for all } \gamma .
$$

To prove this, we first consider types $\gamma$ such that

$$
\rho(q(\gamma)) \geqslant \rho\left(q^{m}\right)+\varepsilon .
$$

Then, by (28), $\tau(q(\gamma))=T(q(\gamma))$. This implies $x(\gamma) \in M^{\prime}$. Thus, by Step 4 and the tie-breaking rule for $x^{\prime}$, we have $x^{\prime}(\gamma)=x(\gamma)$. So (30) holds with equality.

We now consider types $\gamma$ such that

$$
\rho(q(\gamma))<\rho\left(q^{m}\right)+\varepsilon .
$$

Then by (28),

$$
\tau(q(\gamma))=C(q(\gamma))+\rho\left(q^{m}\right)+\varepsilon>T(q(\gamma)) .
$$


Using (28) again,

$$
\tau\left(q^{\prime}(\gamma)\right)-C\left(q^{\prime}(\gamma)\right) \geqslant \rho\left(q^{m}\right)+\varepsilon>\rho(q(\gamma)) .
$$

Step 6 (Final): We prove that $\left(M^{\prime}, x^{\prime}, \Gamma\right)$ generates strictly more profits than $(M, x, \Gamma)$. By (32), it suffices to show that there exists a positive measure of types that satisfy (31). First, note that (31) holds for type $a$ by the definition of $q^{m}$. Let $\gamma_{k}=\hat{\gamma}(k)$. Then (25) implies $\gamma_{k}>a$. By single-crossing, $q(\gamma) \leqslant k$ for all $\gamma<\gamma_{k}$. So, by the definition of $q^{m}$, all those types satisfy (31).

\section{A.4. Proof of Proposition 5}

Suppose, by way of contradiction, that $U(x(b))>0$. By assumption, there exist a type $\gamma \in \Gamma$ and an offer $y \gg x(b)$ such that $\pi(y)>\pi(x(b))$ and $U(y)+V_{\gamma}(y)>U(x(b))+V_{\gamma}(x(b))$. Since $U(x(b))>0$, we can choose $y$ close to $x(b)$ so that $U(y) \geqslant 0$.

We prove that the modified menu $\hat{M} \equiv M \cup\{y\}$ generates larger profits. The associated assignment function is defined by: $\hat{x}(\gamma)=y$ if consumer $\gamma$ strictly prefers $y$ to $x(\gamma)$ in terms of $U+V$ utility, and $\hat{x}(\gamma)=x(\gamma)$ otherwise.

We first show that the schedule $(\hat{M}, \hat{x}, \Gamma)$ satisfies all the incentive constraints. Ex ante IR is preserved since the added item satisfies $U(y) \geqslant 0$. Ex post IC is also satisfied since $y$ is assigned only to consumers who prefer $y$ to what there are initially assigned to. Ex post IR is also satisfied since adding an item makes no one worse off in terms of $U+V$ utility.

So it remains to show that for all types $\gamma$ such that $\hat{x}(\gamma)=y, y$ generates more profits than $x(\gamma)$. Suppose, by contradiction, that there exists $\gamma$ such that $\hat{x}(\gamma)=y$ and $\pi(y) \leqslant \pi(x(\gamma))$. Consider the isoprofit curve through $y$. Then $x(\gamma)$ is above or on the isoprofit curve, while $x(b)$ is below it. By a usual argument of incentive compatibility, $x(\cdot)$ is monotone and so $x(\gamma) \leqslant x(b) \leqslant y$. Let $x^{\prime}=\left(q^{\prime}, t^{\prime}\right)$ be the convex combination of $\{x(\gamma), y\}$ such that $q^{\prime}=q(b)$. Since the technology is convex, $x^{\prime}$ is above or on the isoprofit curve. Since $x(b)$ is below the same curve, it is cheaper than $x^{\prime}$, i.e., $t(b)<t^{\prime}$. Now, recall that $y$ is preferred to $x(\gamma)$ for $U+V_{\gamma}$ since $\hat{x}(\gamma)=y$. Since $U+V_{\gamma}$ is quasi-concave, $x^{\prime}$ is at least as good as $x(\gamma)$ for $U+V_{\gamma}$. Therefore,

$$
U(x(b))+V_{\gamma}(x(b))>U\left(x^{\prime}\right)+V_{\gamma}\left(x^{\prime}\right) \geqslant U(x(\gamma))+V_{\gamma}(x(\gamma)) .
$$

This shows that the initial schedule violates ex post IC for $\gamma$, a contradiction.

\section{A.5. Proof of Proposition 6}

Suppose, by way of contradiction, that there exists an offer $x^{\prime} \geqslant \bar{x}$ such that $U\left(x^{\prime}\right) \geqslant 0$ and $t^{\prime}-C\left(q^{\prime}\right)>\bar{t}-C(\bar{q})$. We claim that the monopolist can earn more profits by offering $M^{\prime}=$ $M \cup\left\{x^{\prime}\right\}$. The argument is similar to that of Proposition 5; just note that $x^{\prime}$ gives a higher utility in $U+V_{\gamma}$ than $\bar{x}$ for all $\gamma^{\prime}>\gamma^{*}$.

\section{A.6. Proof of Proposition 7}

Since $w(\underline{\gamma})=0$ and $w$ is non-decreasing, $w(\gamma)=0$ for all $\gamma \in[a, \underline{\gamma}]$. Therefore, if $\underline{\gamma}>a$, then for all $\gamma \in[a, \underline{\gamma}], v_{2}(q(\gamma), \gamma)=0=q(\gamma)$ and hence the ex ante IR condition (7) binds. By CQ, $\underline{\gamma}<\gamma^{*}$.

We partition $[a, b]$ into two sets:

$$
\begin{aligned}
\Gamma_{+} & \equiv\{\gamma \in[a, b]: w(\gamma) / i>v(q(\gamma), \gamma)-u(q(\gamma))\}, \\
\Gamma_{0} & \equiv\{\gamma \in[a, b]: w(\gamma) / i=v(q(\gamma), \gamma)-u(q(\gamma))\} .
\end{aligned}
$$


Claim 1. For all $\gamma \in \Gamma_{0}$, if $\gamma>\underline{\gamma}$ then $q(\gamma)>0$.

Proof. If $q(\gamma)=0$, then since $\gamma \in \Gamma_{0}, w(\gamma) / i=v(0, \gamma)-u(0)=0$. This implies $\gamma \leqslant \underline{\gamma}$, a contradiction.

Claim 2. $\bar{\gamma} \equiv \sup \Gamma_{+}$satisfies $\bar{\gamma} \geqslant \gamma^{*}$.

Proof. By CQ, $\gamma^{*} \in \Gamma_{+}$, which implies $\gamma^{*} \leqslant \sup \Gamma_{+}=\bar{\gamma}$.

Claim 3. If $\bar{\gamma}<b$, then $q$ is constant over $\bar{I} \equiv[\bar{\gamma}, b] \cap \Gamma_{0}$. (Note that $(\bar{\gamma}, b] \subseteq \Gamma_{0}$ but $\bar{\gamma}$ may not be in $\Gamma_{0}$.)

Proof. Choose any pair $\gamma_{1}, \gamma_{2} \in \bar{I}$ such that $\gamma_{2}>\gamma_{1}$. Since $w^{\prime}=i v_{2}$,

$$
\left[w\left(\gamma_{2}\right)-w\left(\gamma_{1}\right)\right] / i=\int_{\gamma_{1}}^{\gamma_{2}} v_{2}(q(\gamma), \gamma) d \gamma
$$

Since $\gamma_{1}$ and $\gamma_{2}$ belong to $\Gamma_{0}$, the left-hand side of (33) equals

$$
\begin{aligned}
& v\left(q\left(\gamma_{2}\right), \gamma_{2}\right)-u\left(q\left(\gamma_{2}\right)\right)-\left[v\left(q\left(\gamma_{1}\right), \gamma_{1}\right)-u\left(q\left(\gamma_{1}\right)\right)\right] \\
& =\int_{\gamma^{*}}^{\gamma_{2}} v_{2}\left(q\left(\gamma_{2}\right), \gamma\right) d \gamma-\int_{\gamma^{*}}^{\gamma_{1}} v_{2}\left(q\left(\gamma_{1}\right), \gamma\right) d \gamma \\
& =\int_{\gamma^{*}}^{\gamma_{2}} \int_{q\left(\gamma_{1}\right)}^{q\left(\gamma_{2}\right)} v_{12}(q, \gamma) d q d \gamma+\int_{\gamma_{1}}^{\gamma_{2}} v_{2}\left(q\left(\gamma_{1}\right), \gamma\right) d \gamma .
\end{aligned}
$$

Thus (33) reduces to

$$
\int_{\gamma^{*}}^{\gamma_{2}} \int_{q\left(\gamma_{1}\right)}^{q\left(\gamma_{2}\right)} v_{12}(q, \gamma) d q d \gamma=\int_{\gamma_{1}}^{\gamma_{2}} \int_{q\left(\gamma_{1}\right)}^{q(\gamma)} v_{12}(q, \gamma) d q d \gamma,
$$

which is equivalent to

$$
\int_{\gamma^{*}}^{\gamma_{1}} \int_{q\left(\gamma_{1}\right)}^{q\left(\gamma_{2}\right)} v_{12}(q, \gamma) d q d \gamma+\int_{\gamma_{1}}^{\gamma_{2}} \int_{q(\gamma)}^{q\left(\gamma_{2}\right)} v_{12}(q, \gamma) d q d \gamma=0
$$

This equation holds for any $\gamma_{1}, \gamma_{2} \in \bar{I}$ such that $\gamma_{1}<\gamma_{2}$.

To prove that $q$ is constant over $\bar{I}$, suppose, by way of contradiction, that there exists $\gamma_{3}, \gamma_{4} \in \bar{I}$ such that $\gamma_{3}<\gamma_{4}$ and $q\left(\gamma_{3}\right) \neq q\left(\gamma_{4}\right)$.

We first consider the case when $q\left(\gamma_{3}\right)<q\left(\gamma_{4}\right)$. Let $q^{\prime}=\sup \left\{q(\gamma): \gamma_{3} \leqslant \gamma \leqslant \gamma_{4}\right\}$. Then there exists a convergent sequence $\left\{\gamma^{k}\right\}$ such that $\gamma^{k} \in\left[\gamma_{3}, \gamma_{4}\right]$ and $q\left(\gamma^{k}\right) \rightarrow q^{\prime}$. By applying (34) to $\gamma_{1}=\gamma_{3}$ and $\gamma_{2}=\gamma^{k}$, we get

$$
\int_{\gamma^{*}}^{\gamma_{3}} \int_{q\left(\gamma_{3}\right)}^{q\left(\gamma^{k}\right)} v_{12}(q, \gamma) d q d \gamma+\int_{\gamma_{3}}^{\gamma^{k}} \int_{q(\gamma)}^{q\left(\gamma^{k}\right)} v_{12}(q, \gamma) d q d \gamma=0 .
$$


Since this holds for all $k$, we can take the limit. Let $\gamma^{\prime} \in\left[\gamma_{3}, \gamma_{4}\right]$ denote the limit of $\left\{\gamma^{k}\right\}$. Then

$$
\begin{aligned}
0 & =\int_{\gamma^{*}}^{\gamma_{3}} \int_{q\left(\gamma_{3}\right)}^{q^{\prime}} v_{12}(q, \gamma) d q d \gamma+\int_{\gamma_{3}}^{\gamma^{\prime}} \int_{q(\gamma)}^{q^{\prime}} v_{12}(q, \gamma) d q d \gamma \\
& \geqslant \int_{\gamma^{*}}^{\gamma_{3}} \int_{q\left(\gamma_{3}\right)}^{q^{\prime}} v_{12}(q, \gamma) d q d \gamma>0,
\end{aligned}
$$

which is a contradiction.

If $q\left(\gamma_{3}\right)>q\left(\gamma_{4}\right)$, a symmetric argument works. Specifically, if we set $q^{\prime}=\inf \left\{q(\gamma): \gamma_{3} \leqslant \gamma \leqslant \gamma_{4}\right\}$, we obtain (35) with reverse inequalities.

Let $\bar{q} \equiv q(b)$.

Claim 4. $\bar{\gamma}>\gamma^{*}$.

Proof. We already proved $\bar{\gamma} \geqslant \gamma^{*}$. Suppose, by way of contradiction, that $\bar{\gamma}=\gamma^{*}$. We have noted $(\bar{\gamma}, b] \subseteq \Gamma_{0}$ and the previous claim says $q(\gamma)=\bar{q}$ over the interval. Thus, taking the limit as $\gamma \downarrow \bar{\gamma}$ gives

$$
w\left(\gamma^{*}\right) / i=v\left(\bar{q}, \gamma^{*}\right)-u(\bar{q})=0
$$

since $v\left(\cdot, \gamma^{*}\right)=u(\cdot)$. On the other hand, since $\gamma^{*} \in \Gamma_{+}$(by CQ),

$$
w\left(\gamma^{*}\right) / i>v\left(q\left(\gamma^{*}\right), \gamma^{*}\right)-u\left(q\left(\gamma^{*}\right)\right)=0 .
$$

This is a contradiction with (36).

Claim 5. $\lambda(\bar{\gamma})=0$.

Proof. If $\bar{\gamma} \in \Gamma_{+}$, the result is obvious from (11). So, suppose $\bar{\gamma} \in \Gamma_{0}$. Then $q(\bar{\gamma})=\bar{q}$ and Claim 1 implies $\bar{q}>0$. Since $q$ is continuous from the left, $q(\gamma)>0$ for all $\gamma$ close to $\bar{\gamma}$. The definition of $\bar{\gamma}$ implies that for all $\varepsilon>0$, there exists $\gamma \in(\bar{\gamma}-\varepsilon, \bar{\gamma})$ such that $\gamma \in \Gamma_{+}$and so $\lambda(\gamma)=0$. Therefore, taking the limit of (9) as $\gamma \uparrow \bar{\gamma}$, we get

$$
\left[u^{\prime}(\bar{q})+i v_{1}(\bar{q}, \bar{\gamma})-(1+i) C^{\prime}(\bar{q})\right] f(\bar{\gamma})+\mu(\bar{\gamma}) i v_{12}(\bar{q}, \bar{\gamma})=0 .
$$

On the other hand, evaluating (9) at $\bar{\gamma}$ yields

$$
\left[u^{\prime}(\bar{q})+i v_{1}(\bar{q}, \bar{\gamma})-(1+i) C^{\prime}(\bar{q})\right] f(\bar{\gamma})+\mu(\bar{\gamma}) i v_{12}(\bar{q}, \bar{\gamma})+\lambda(\bar{\gamma}) i\left[u^{\prime}(\bar{q})-v_{1}(\bar{q}, \bar{\gamma})\right]=0 .
$$

Thus, the last term is zero. Since $\bar{\gamma}>\gamma^{*}$ and $\bar{q}>0$, we have $u^{\prime}(\bar{q})<v_{1}(\bar{q}, \bar{\gamma})$ and hence $\lambda(\bar{\gamma})=0$.

Claim 6. If $\bar{\gamma}<b$, then $\mu(\bar{\gamma})<0$.

Proof. Since $(\bar{\gamma}, b] \subseteq \Gamma_{0}$, taking the limit as $\gamma \downarrow \bar{\gamma}$ gives

$$
v(\bar{q}, \bar{\gamma})-u(\bar{q})=w(\bar{\gamma}) / i \geqslant v(q(\bar{\gamma}), \bar{\gamma})-u(q(\bar{\gamma})) .
$$

Since $\bar{\gamma}>\gamma^{*}$, the inequality implies

$$
q(\bar{\gamma}) \leqslant \bar{q} .
$$


By Claim 1, $\bar{q}>0$. By (9) and (10), for all $\gamma>\bar{\gamma}$,

$$
(1+i)\left[u^{\prime}(\bar{q})-C^{\prime}(\bar{q})\right] f(\gamma)+\mu(\gamma) i v_{12}(\bar{q}, \gamma)-\mu^{\prime}(\gamma) i\left[u^{\prime}(\bar{q})-v_{1}(\bar{q}, \gamma)\right]=0 .
$$

We can solve the differential equation by using the fact that the sum of the last two terms is the derivative of $-\mu(\gamma) i\left[u^{\prime}(\bar{q})-v_{1}(\bar{q}, \gamma)\right]$, together with the boundary condition $\mu(b)=0$ (see (12)). This gives

$$
\mu(\bar{\gamma})=\frac{1+i}{i} \frac{(1-F(\bar{\gamma}))\left[u^{\prime}(\bar{q})-C^{\prime}(\bar{q})\right]}{v_{1}(\bar{q}, \bar{\gamma})-u^{\prime}(\bar{q})} .
$$

Since $\lambda(\bar{\gamma})=0$, Eq. (9) evaluated at $\bar{\gamma}$ yields

$$
\left[u^{\prime}(q(\bar{\gamma}))+i v_{1}(q(\bar{\gamma}), \bar{\gamma})-(1+i) C^{\prime}(q(\bar{\gamma}))\right] f(\bar{\gamma})+\mu(\bar{\gamma}) i v_{12}(q(\bar{\gamma}), \bar{\gamma})+\delta(\bar{\gamma})=0 .
$$

Now, suppose, by way of contradiction, that $\mu(\bar{\gamma}) \geqslant 0$. Then (39) implies

$$
u^{\prime}(q(\bar{\gamma}))+i v_{1}(q(\bar{\gamma}), \bar{\gamma}) \leqslant(1+i) C^{\prime}(q(\bar{\gamma})) .
$$

On the other hand, (38) implies $u^{\prime}(\bar{q}) \geqslant C^{\prime}(\bar{q})$. Since $\bar{\gamma}>\gamma^{*}$ and $v_{12}>0$, this implies

$$
u^{\prime}(\bar{q})+i v_{1}(\bar{q}, \bar{\gamma})>(1+i) C^{\prime}(\bar{q}) .
$$

This and (40) imply $q(\bar{\gamma})>\bar{q}$, which is a contradiction with (37).

Claim 7. $\mu(\gamma) \leqslant 0$ for all $\gamma<\bar{\gamma}$ that is sufficiently close to $\bar{\gamma}$.

Proof. Since $\mu$ is continuous, the result follows trivially from Claim 6 if $\bar{\gamma}<b$. So, suppose $\bar{\gamma}=b$. Since $\mu^{\prime}(b)=f(b)-\lambda(b)$ and Claim 5 implies $\lambda(b)=0$, we obtain $\mu^{\prime}(b)=f(b) \geqslant 0$. This and $\mu(b)=0$ imply the desired result.

By Claim 7, there exists $\varepsilon>0$ such that $\mu(\gamma) \leqslant 0$ for all $\gamma \in[\bar{\gamma}-\varepsilon, \bar{\gamma}]$. In what follows, let $\tilde{\gamma} \in[\bar{\gamma}-\varepsilon, \bar{\gamma}]$ be such that $\tilde{\gamma} \in \Gamma_{+}$. Let $\tilde{I}$ be the maximal interval such that $\tilde{\gamma} \in \tilde{I}$ and $\tilde{I} \subseteq \Gamma_{+}$.

Claim 8. $q$ is non-decreasing over $\tilde{I}$.

Proof. Define a function $\beta:[a, b] \rightarrow \mathbb{R}$ by

$$
\beta(\gamma) \equiv F(\gamma)-\mu(\gamma)
$$

Since $\beta^{\prime}(\gamma)=\lambda(\gamma)$, it follows that over the interval $\tilde{I}, \beta(\gamma)$ is constant and equal to $\beta(\tilde{\gamma})$. Since $\mu(\tilde{\gamma}) \leqslant 0, \beta(\tilde{\gamma}) \geqslant 0$. Since $\beta$ is globally non-decreasing, $\beta(\tilde{\gamma}) \leqslant \beta(b)=1$. Eq. (9) implies that for all $\gamma \in \tilde{I}$,

$$
u^{\prime}(q(\gamma))+i v_{1}(q(\gamma), \gamma)-i v_{12}(q(\gamma), \gamma) \frac{\beta(\tilde{\gamma})-F(\gamma)}{f(\gamma)}+\frac{\delta(\gamma)}{f(\gamma)}=(1+i) C^{\prime}(q(\gamma)) .
$$

Define a function $B: \mathbb{R}_{+} \times[a, b] \rightarrow \mathbb{R}$ by

$$
B(q, \gamma) \equiv u(q)+i v(q, \gamma)-i v_{2}(q, \gamma) \frac{\beta(\tilde{\gamma})-F(\gamma)}{f(\gamma)}-(1+i) C(q)
$$


Then for all $\gamma \in \tilde{I}, q(\gamma)$ satisfies $B_{q}(q(\gamma), \gamma) \leqslant 0$ and $q(\gamma) B_{q}(q(\gamma), \gamma)=0$. For all $\gamma \in \tilde{I}$, $\beta(\tilde{\gamma})-F(\gamma)=-\mu(\gamma) \geqslant 0$. Since we assume $C^{\prime \prime}>0$ and $v_{112} \geqslant 0$,

$$
B_{q q}=u^{\prime \prime}(q, \gamma)+i v_{11}(q, \gamma)-i v_{112}(q, \gamma) \frac{\beta(\tilde{\gamma})-F(\gamma)}{f(\gamma)}-(1+i) C^{\prime \prime}(q)<0 .
$$

Thus, $q(\gamma)$ is determined uniquely. A sufficient condition for $q$ to be strictly increasing is $B_{q \gamma}>0$. The cross derivative is

$$
B_{q \gamma}=i v_{12}(q, \gamma)-i v_{12}(q, \gamma) \frac{\partial\left(\frac{\beta(\tilde{\gamma})-F(\gamma)}{f(\gamma)}\right)}{\partial \gamma}-i v_{122}(q, \gamma) \frac{\beta(\tilde{\gamma})-F(\gamma)}{f(\gamma)} .
$$

Since $\beta(\tilde{\gamma})-F(\gamma) \geqslant 0$ and we assume $v_{122} \leqslant 0<v_{12}$, a sufficient condition for $B_{q \gamma}>0$ is that $(\beta(\tilde{\gamma})-F(\gamma)) / f(\gamma)$ is non-increasing. This is indeed the case since $\beta(\tilde{\gamma}) \in[0,1]$ and $f$ is $\log$ concave [2].

Define a function $h: \mathbb{R}_{+} \times[a, b] \rightarrow \mathbb{R}$ by

$$
h(q, \gamma) \equiv w(\gamma)-i v(q, \gamma)+i u(q) .
$$

Claim 9. $h(q(\gamma), \gamma)$ is non-increasing in $\gamma$ over $\tilde{I} \cap\left[\gamma^{*}, b\right]$.

Proof. Let $\gamma, \gamma^{\prime} \in \tilde{I}$ be such that $\gamma>\gamma^{\prime} \geqslant \gamma^{*}$. Since $q$ is non-decreasing over the interval,

$$
\begin{aligned}
h\left(q\left(\gamma^{\prime}\right), \gamma^{\prime}\right) & =w\left(\gamma^{\prime}\right)-i v\left(q\left(\gamma^{\prime}\right), \gamma^{\prime}\right)+i u\left(q\left(\gamma^{\prime}\right)\right) \\
& \geqslant w\left(\gamma^{\prime}\right)-i v\left(q(\gamma), \gamma^{\prime}\right)+i u(q(\gamma)) .
\end{aligned}
$$

Now, for all $\theta \in\left[\gamma^{\prime}, \gamma\right]$, monotonicity over $\tilde{I}$ implies $q(\theta) \leqslant q(\gamma)$. Since $v_{12} \geqslant 0$, we get $v_{2}(q(\theta), \theta)$ $\leqslant v_{2}(q(\gamma), \theta)$. Since $w^{\prime}=i v_{2}$, the inequality implies that $w(\theta)-i v(q(\gamma), \theta)$ is non-increasing in $\theta$ over $\left[\gamma^{\prime}, \gamma\right]$. Thus

$$
w\left(\gamma^{\prime}\right)-i v\left(q(\gamma), \gamma^{\prime}\right)+i u(q(\gamma)) \geqslant w(\gamma)-i v(q(\gamma), \gamma)+i u(q(\gamma))=h(q(\gamma), \gamma) .
$$

This and (42) prove the desired result.

$$
\text { Let } \underset{\sim}{\gamma} \equiv \inf \tilde{I} \text { and } \underset{\sim}{q} \equiv \lim _{\gamma \downarrow \underset{\sim}{\gamma}} q(\gamma)
$$

Claim 10. $\underset{\sim}{\gamma}<\gamma^{*}$

Proof. Suppose, by way of contradiction, that $\underset{\gamma}{\gamma} \geqslant \gamma^{*}$. The definition of $\tilde{I}$ implies $\tilde{I} \subseteq \Gamma_{+}$and hence $h(q(\gamma), \gamma)>0$ over the interval. Since $h \tilde{(q}(\cdot), \cdot)$ is non-increasing, taking the limit yields $h(q, \gamma)>0$, i.e., $w(\gamma) / i>v\left(q, \gamma^{\gamma}\right)-u(q)$. On the other hand, the maximality of $\tilde{I}$ implies that, within any neighborhood of $\gamma$, there exists $\gamma<\gamma$ such that $\gamma \in \Gamma_{0}$. Since $q$ is continuous from the left, taking the limit yields $\tilde{w}(\underset{\sim}{\gamma}) / i=v(q(\underset{\sim}{\gamma}), \underset{\sim}{\gamma})-u(q(\underset{\sim}{\gamma}))$. Thus

$$
v(q(\underset{\sim}{\gamma}), \underset{\sim}{\gamma})-u(q(\underset{\sim}{\gamma}))>v(\underset{\sim}{q}, \underset{\sim}{\gamma})-u(\underset{\sim}{q})
$$

Since $\underset{\sim}{\gamma} \geqslant \gamma^{*}$, the inequality implies

$$
q(\underset{\sim}{\gamma})>\underset{\sim}{q} .
$$


Since (41) holds for all $\gamma \in \tilde{I}$, taking the limit yields

$$
u^{\prime}(\underset{q}{q})+i v_{1}(\underset{\sim}{q}, \underset{\sim}{\gamma})-i v_{12}(\underset{\sim}{q}, \underset{\sim}{\gamma}) \frac{\beta(\tilde{\gamma})-F(\underset{\sim}{\gamma})}{f(\underset{\sim}{\gamma})} \leqslant(1+i) C^{\prime}(\underset{\sim}{q}) .
$$

By $(43), q(\underset{\sim}{\gamma})>0$. Thus, evaluating (9) at $\underset{\sim}{\gamma}$ yields

$$
\begin{aligned}
& \left.u^{\prime}(q(\underset{\sim}{\gamma}))+i v_{1}(q(\underset{\sim}{\gamma}), \underset{\sim}{\gamma})-i v_{12}(q \underset{\sim}{\gamma}), \underset{\sim}{\gamma}\right) \frac{\beta(\tilde{\gamma})-F(\underset{\sim}{\gamma})}{f(\underbrace{\gamma}_{\sim})} \\
& \quad+\lambda(\underset{\sim}{\gamma}) i\left[u^{\prime}(q(\underset{\sim}{\gamma}))-v_{1}(q(\underset{\sim}{\gamma}), \underset{\sim}{\gamma})\right] / f(\underset{\sim}{\gamma})=(1+i) C^{\prime}(q(\underset{\sim}{\gamma})) .
\end{aligned}
$$

Since $\gamma \geqslant \gamma^{*}, u^{\prime}(q(\gamma))-v_{1}(q(\gamma), \gamma) \leqslant 0$. Thus (45) implies $B_{q}(q(\gamma), \gamma) \geqslant 0$, while (44) says $B_{q}(\underset{\sim}{q}, \underset{\sim}{\tilde{\gamma}}) \leqslant 0$. Since $B_{q q}<0$, the inequalities imply $q(\underset{\sim}{\gamma}) \leqslant \underset{\sim}{q}$, which is a contradiction with (43).

Remark 1. Claims 8-10 are proved for any $\tilde{\gamma}<\bar{\gamma}$ that is close to $\bar{\gamma}$ (so that $\mu(\tilde{\gamma}) \leqslant 0$ ) and such that $\tilde{\gamma} \in \Gamma_{+}$. Thus, Claim 10 implies that $\tilde{I}$ and $\gamma$ are the same for all these $\tilde{\gamma}$. This implies sup $\tilde{I}=\bar{\gamma}$. Moreover, $q$ is non-decreasing over $\tilde{I} \cup \bar{I}$ by Claim 8, (37), and Claim 3.

Claim 11. $\underset{\sim}{\gamma}=\underline{\gamma}$.

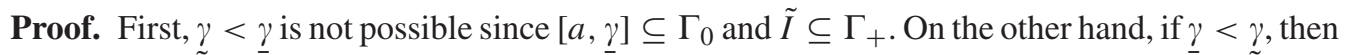
the definition of $\bar{\gamma}$ implies that there exists $\gamma \in(\underline{\gamma}, \gamma)$ such that $\gamma \in \Gamma_{0}$. But then, $0 \leqslant \overline{\leqslant}(\tilde{\gamma}) / i=$ $v(q(\gamma), \gamma)-u(q \tilde{(\gamma)}) \leqslant 0$ since $\gamma<\gamma^{*}$. Therefore $\tilde{w} \tilde{(\gamma)}=0$, which is a contradiction with $\gamma>\underline{\gamma}$.

Proposition 7 can now be proved as follows:

For the monotonicity of $q$, if $\tilde{I} \cup \bar{I}=[a, b]$, then it follows from the last statement in Remark 1. If $\underline{\gamma}>a$, it suffices to note that $q(\gamma)=0$ over $[a, \gamma]$. The only remaining case to be considered is when $\underline{\gamma}=a$ and $a \in \Gamma_{0}$. In this case, $0=w(a) / i=v(q(a), a)-u(q(a)) \leqslant 0$ and hence $q(a)=0$ and the monotonicity follows.

Statement 1 is proved in Claim 3.

Eq. (15) comes from (41). But a few things need to be proved. First, for $\gamma>\underline{\gamma}$, we need to show $q(\gamma)>0$. If $q(\gamma)=0$ for some $\gamma>\underline{\gamma}$, the monotonicity of $q$ implies $q\left(\gamma^{\prime}\right)=w\left(\gamma^{\prime}\right)=0$ for all $\gamma^{\prime} \in[a, \gamma]$, which is a contradiction with $\gamma>\underline{\gamma}$.

To prove (15) for $\gamma=\underline{\gamma}$, we take the limit of (41) as $\gamma \downarrow \underline{\gamma}$. This gives

$$
u^{\prime}(\underset{\sim}{q})+i v_{1}(\underset{\sim}{q}, \underline{\gamma})-i v_{12}(\underset{\sim}{q}, \underline{\gamma}) \frac{\beta(\bar{\gamma})-F(\underline{\gamma})}{f(\underline{\gamma})}=(1+i) C^{\prime}(\underset{q}{q}) .
$$

On the other hand, evaluating (9) at $\underline{\gamma}$ yields

$$
\begin{aligned}
& u^{\prime}(q(\underline{\gamma}))+i v_{1}(q(\underline{\gamma}), \underline{\gamma})-i v_{12}(q(\underline{\gamma}), \underline{\gamma}) \frac{\beta(\bar{\gamma})-F(\underline{\gamma})}{f(\underline{\gamma})} \\
& +\frac{\lambda(\underline{\gamma}) i\left[u^{\prime}(q(\underline{\gamma}))-v_{1}(q(\underline{\gamma}), \underline{\gamma})\right]}{f(\underline{\gamma})}+\frac{\delta(\underline{\gamma})}{f(\underline{\gamma})}=(1+i) C^{\prime}(q(\underline{\gamma})) .
\end{aligned}
$$


Since $\underline{\gamma}<\gamma^{*}, u^{\prime}(q)-v_{1}(q, \underline{\gamma}) \geqslant 0$. Thus (47) implies $B_{q}(q(\underline{\gamma}), \underline{\gamma}) \leqslant 0$, whereas (46) says $B_{q}(q, \underline{\gamma})=$ 0 . Since $B_{q q}<0$ and $q$ is monotonic, $q(\underline{\gamma})=\underset{q}{q}$. Substituting this into (46) yields (15) for $\underline{\gamma}$.

\section{A.7. Proof of Proposition 9}

Suppose, by way of contradiction, that $\underline{\gamma} \geqslant \underline{\gamma}^{\mathrm{S}}$ and $\underline{\gamma}>a$. The latter inequality implies $q(\underline{\gamma})=0$. Then

$$
B_{q}(0, \underline{\gamma})=0 \geqslant B_{q}\left(0, \underline{\gamma}^{\mathrm{S}}\right) \geqslant B_{q}\left(q^{\mathrm{S}}\left(\underline{\gamma}^{\mathrm{S}}\right), \underline{\gamma}^{\mathrm{S}}\right)=i v_{12}\left(q^{\mathrm{S}}\left(\underline{\gamma}^{\mathrm{S}}\right), \underline{\gamma}^{\mathrm{S}}\right) \frac{1-\beta(\bar{\gamma})}{f\left(\underline{\gamma}^{\mathrm{S}}\right)}>0,
$$

which is a contradiction.

\section{A.8. Proof of Proposition 10}

It remains to consider the case where $\gamma>\bar{\gamma}$ or $\gamma \leqslant \underline{\gamma}$. First, consider the case where $\gamma \leqslant \underline{\gamma}$. Since $q>0, \gamma=\underline{\gamma}=a$. Eq. (15) then says $B_{q}(q, a)=0$. Similarly, since $q>0, \gamma^{\mathrm{S}} \geqslant \underline{\gamma}^{\mathrm{S}}$. The equality $\gamma^{\mathrm{S}}=\underline{\gamma}^{\mathrm{S}}$ holds only if $\gamma^{\mathrm{S}}=a$. By (17),

$$
B_{q}\left(q, \gamma^{\mathrm{S}}\right)=i v_{12}\left(q, \gamma^{\mathrm{S}}\right) \frac{1-\beta(\bar{\gamma})}{f\left(\gamma^{\mathrm{S}}\right)}>0=B_{q}(q, a) .
$$

The inequality implies $\gamma^{\mathrm{S}}>a$. Therefore, $\gamma^{\mathrm{S}}>\underline{\gamma}^{\mathrm{S}}$.

Let $B^{\mathrm{S}}(q, t)$ be defined by replacing $\beta(\bar{\gamma})$ with 1 in (19). With the definition, (17) says $B^{\mathrm{S}}\left(q^{\mathrm{S}}(\gamma), \gamma\right)=0$ for all $\gamma \geqslant \underline{\gamma}^{\mathrm{S}}$. The argument we used for $B(q, t)$ can be used to show $B_{q q}^{\mathrm{S}}<0$ and $B_{q \gamma}^{\mathrm{S}}>0$. Thus $q^{\mathrm{S}}(\gamma)$ is strictly increasing for $\gamma \geqslant \underline{\gamma}^{\mathrm{S}}$. This and $\gamma^{\mathrm{S}}>\underline{\gamma}^{\mathrm{S}}$ imply

$$
\begin{aligned}
U(q, t)+V_{\gamma \mathrm{S}}(q, t) & =u(q)-t+i\left[v\left(q, \gamma^{\mathrm{S}}\right)-t\right. \\
& =U(q, t)+V_{a}(q, t)+\int_{a}^{\gamma^{\mathrm{S}}} i v_{2}(q, y) d y \\
& >\int_{a}^{\gamma^{\mathrm{S}}} i v_{2}\left(q^{\mathrm{S}}(y), y\right) d y=w^{\mathrm{S}}\left(\gamma^{\mathrm{S}}\right)=U\left(q, t^{\mathrm{S}}\right)+V_{\gamma^{\mathrm{S}}}\left(q, t^{\mathrm{S}}\right) .
\end{aligned}
$$

The inequality implies $t<t^{\mathrm{S}}$.

Lastly, consider the case in which $\gamma>\bar{\gamma}$. Thus $(q, t)=x(b)$. This case is relevant only if $x(\cdot)$ has a discontinuity at $\bar{\gamma}$ (from the right) so that $x(\bar{\gamma})<x(b)$. It is easy to show that type $\bar{\gamma}$ is indifferent between $x(\bar{\gamma})$ and $x(b)$ in terms of $U+V_{\bar{\gamma}}$. Therefore, $U(q, t)+V_{\bar{\gamma}}(q, t)=w(\bar{\gamma})$ and so we obtain (21) where $\gamma$ is replaced with $\bar{\gamma}$.

\section{References}

[1] J. Ameriks, A. Caplin, J. Leahy, T. Tyler, Measuring self control, mimeo. New York University, 2003.

[2] M. Bagnoli, T. Bergstrom, Log-concave probability and its applications, Econ. Theory 26 (2005) 445-469.

[3] D. Besanko, S. Donnenfeld, L.J. White, Monopoly and quality distortion: effects and remedies, Quart. J. Econ. 102 (1987) 743-767.

[4] K. Chatterjee, R. V. Krishna, Menu choice, environmental cues and temptation: a "dual self" approach to self-control, mimeo, 2005.

[5] D. DeJong, M. Ripoll, Do self-control preferences help explain the puzzling behavior of asset prices? J. Monet. Econ., 2006, forthcoming. 
[6] E. Dekel, B. L. Lipman, A. Rustichini, Temptation-driven preferences, mimeo, 2005.

[7] S. DellaVigna, U. Malmendier, Contract design and self-control: theory and evidence, Quart. J. Econ. 119 (2004) 353-402.

[8] S. DellaVigna, U. Malmendier, Paying not to go to the gym, Amer. Econ. Rev. 96 (2006) 694-719.

[9] S. Esteban, E. Miyagawa, Optimal menu of menus with self-control preferences, mimeo, 2005.

[10] S. Esteban, E. Miyagawa, Temptation, self-control, and competitive nonlinear pricing, Econ. Lett. 90 (2006) 348-355.

[11] D. Fudenberg, D.K. Levine, A dual self model of impulse control, mimeo, 2005.

[12] D. Fudenberg, J. Tirole, Game Theory, MIT Press, Cambridge, MA, 1992.

[13] S.M. Gilpatric, Present-biased preferences, self-awareness and shirking in a principal-agent setting, mimeo, University of Tennessee, 2003.

[14] F. Gul, W. Pesendorfer, Temptation and self-control, Econometrica 69 (2001) 1403-1436.

[15] P. Krusell, B. Kuruşcu, A. Smith, Temptation and taxation, mimeo, University of Rochester, 2000.

[16] P. Krusell, B. Kuruşcu, A. Smith, Time orientation and asset prices, J. Monet. Econ. 49 (2002) 107-135.

[17] E. Maskin, J. Riley, Monopoly with incomplete information, RAND J. Econ. 15 (1984) 171-196.

[18] E. Miravete, Choosing the wrong calling plan? ignorance and learning, Amer. Econ. Rev. 93 (2003) 297-310.

[19] M. Mussa, S. Rosen, Monopoly and product quality, J. Econ. Theory 18 (1978) 301-317.

[20] T. O'Donoghue, M. Rabin, Incentives for procrastinators, Quart. J. Econ. 114 (1999) 769-816.

[21] T.C. Schelling, Egonomics, or the art of self-management, Amer. Econ. Rev. 68 (1978) 290-294.

[22] A. Seierstad, K. Sydsæter, Optimal Control Theory with Economic Applications, North-Holland, Amsterdam, 1987.

[23] H.M. Shefrin, R.H. Thaler, The behavioral life-cycle hypothesis, Econ. Inquiry 26 (1988) 609-643.

[24] R.H. Thaler, H.M. Shefrin, An economic theory of self-control, J. Polit. Economy 89 (1981) 392-406.

[25] K. Wertenbroch, Consumption and self-control by rationing purchase quantities of virtue and vice, Marketing Sci. 17 (1998) 317-337.

[26] G.C. Winston, Addiction and backsliding: a theory of compulsive consumption, J. Econ. Behav. Organ. 1 (1980) 295-324. 